Check for updates

Cite this: RSC Adv., 2021, 11, 32750

\title{
Biomedical nanobubbles and opportunities for microfluidics
}

\author{
Ali A. Paknahad, ${ }^{\text {abc }}$ Liam Kerr, $^{\text {abc }}$ Daniel A. Wong, (D) bce Michael C. Kolios (D) bcd \\ and Scott S. H. Tsai (D) *abcf
}

The use of bulk nanobubbles in biomedicine is increasing in recent years, which is attributable to the array of therapeutic and diagnostic tools promised by developing bulk nanobubble technologies. From cancer drug delivery and ultrasound contrast enhancement to malaria detection and the diagnosis of acute donor tissue rejection, the potential applications of bulk nanobubbles are broad and diverse. Developing these technologies to the point of clinical use may significantly impact the quality of patient care. This review compiles and summarizes a representative collection of the current applications, fabrication techniques, and characterization methods of bulk nanobubbles in biomedicine. Current state-of-the-art generation methods are not designed to create nanobubbles of high concentration and low polydispersity, both characteristics of which are important for several bulk nanobubble applications. To date, microfluidics has not been widely considered as a tool for generating nanobubbles, even though the small-scale precision and real-time control offered by microfluidics may overcome the challenges mentioned above. We suggest possible uses of microfluidics for improving the quality of bulk nanobubble populations and propose ways of leveraging existing microfluidic technologies, such as organ-on-a-chip platforms, to expand the experimental toolbox of researchers working to develop biomedical nanobubbles.

Received 24th June 2021

Accepted 19th September 2021

DOI: $10.1039 / \mathrm{d} 1 \mathrm{ra0} 4890 \mathrm{~b}$

rsc.li/rsc-advances found in liquid suspension that have a diameter less than $1000 \mathrm{~nm}$, and are subjected to Brownian motion., ${ }^{\mathbf{4} 5}$ Our review focuses on bulk nanobubbles, detailing their characterization, production and applications in biomedicine. We also attempt to provide insights and guidance for future microfluidics research in this emerging field.

Historically, the earliest evidence of bulk nanobubbles is from a 1981 report by Johnson and Cooke. ${ }^{1}$ They describe the production of submicron gas-filled bubbles due to shear stress in seawater. The authors also found that the bulk nanobubbles exhibit stability for prolonged periods $(>22 \mathrm{~h}) .{ }^{1}$ Since then, there have been many questions that challenge the stability and the existence of bulk nanobubbles post-production. Some of the reluctance to accept the existence of stable bulk nanobubbles stems from predictions of theoretical models that predict a vanishingly short lifetime for nanobubbles. A primary factor considered is based on the capillarity model of Young and Laplace, which states that the pressure inside a bubble is greater than the pressure outside the bubble by an amount dictated by the gas-liquid surface tension and the bubble radius, a phenomenon known as Laplace pressure, ${ }^{6}$

$$
\Delta P_{\text {Laplace }}=P_{\text {Inside }}-P_{\text {Outside }}=\frac{2 \gamma}{r},
$$

where $P_{\text {Inside }}$ and $P_{\text {Outside }}$ are the pressure inside and outside of the bubble, respectively. Also, $\gamma$ is the gas-liquid surface tension, and $r$ is the bubble radius. For sub-micron bubbles, 
such as bulk nanobubbles, where the radius is in the nanometer range, the pressure inside the bubble is predicted to be very high. From here, Henry's law for the solubility of a gas in an aqueous phase is used to explain the destabilization of the bubble in response to the large pressure gradient across the bubble shell. As a result, bubbles can either (a) increase in size by inward diffusion of gas from the surrounding and then be removed from solution by buoyant forces, or (b) they can shrink through outward diffusion, eventually disappearing once all the gas molecules have been removed from the bubble. ${ }^{7}$

Epstein and Plesset, pioneers in understanding bubble growth and dissolution, observed this phenomenon and developed a mathematical model for the diffusion process around a bubble in which they state, "a gas bubble in a liquidgas solution will grow or shrink by diffusion accordingly as the solution is oversaturated or undersaturated." ${ }^{8}$ They estimate the time-dependent bubble radius,

$$
r(t) \approx \sqrt{r_{\mathrm{o}}^{2}+2 \alpha t},
$$

where, $r_{\mathrm{o}}$ is the initial bubble radius, $t$ is time, and $\alpha$ is a positive constant given by, $\frac{D\left(C_{\mathrm{i}}-C_{\mathrm{S}}\right)}{\rho} . D$ is the diffusion coefficient of the gas at the liquid interface, $C_{\mathrm{s}}$ is the concentration of dissolved gas immediately adjacent to the bubble, $C_{\mathrm{i}}$ is the initial concentration of dissolved gas in solution, and $\rho$ is the density of the gas within the bubble. ${ }^{\mathbf{8}}$ The theory suggests that bubbles suspended in a gas-saturated solution rapidly shrink and vanish, as shown by Alheshibri et al. in Fig. $1 .^{9}$ Epstein and Plesset's theory predicts that a bubble whose diameter is less than $1000 \mathrm{~nm}$ has a lifetime that is less than $0.02 \mathrm{~s}$, a time too short to be measured or detected. ${ }^{9}$ Despite these theoretical predictions, experimental evidence over the last decade, particularly the notable works by Nirmalkar et al., have demonstrated the existence and stability of bulk nanobubbles. ${ }^{9-13}$ We note that this review is not focused on the debate regarding the existence and stability of bulk nanobubbles, and readers are encouraged to read the work of Alhesibri et al. titled "A History of Nanobubbles" for an extensive description of the subject of bulk nanobubble stability. ${ }^{9}$

Briefly, several theories attempt to explain the unexpected stability of bulk nanobubbles. One explanation is that

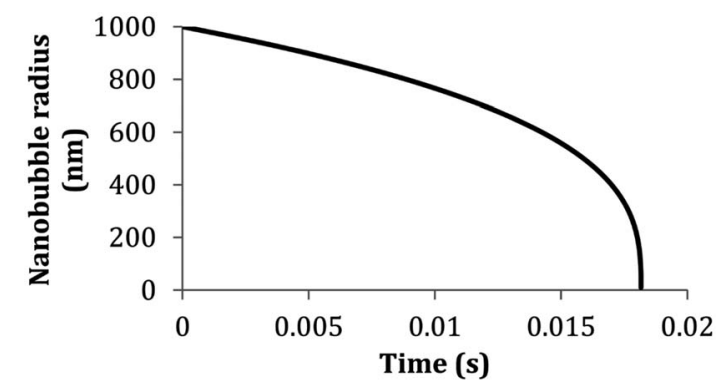

Fig. 1 Lifetime of a bubble smaller than $1000 \mathrm{~nm}$ predicted by the theory of Epstein and Plesset. The graph depicts the calculated radius of a nitrogen-filled bubble in a nitrogen saturated solution versus time $\left(T=300 \mathrm{~K}, \gamma=0.072 \mathrm{~J} \mathrm{~m}^{-2}, D=2.0 \times 10^{-9} \mathrm{~m}^{2} \mathrm{~s}^{-1}, C_{\text {sat }}=0.6379 \mathrm{~mol}\right.$ $\left.\mathrm{m}^{-3}, \rho_{1 \mathrm{~atm}}=40.6921 \mathrm{~mol} \mathrm{~m}^{-3}\right)^{8,9}$ nanobubble stability is attributed to their low rising velocity, which is almost negligible due to their strong Brownian effect, and a low level of buoyancy. ${ }^{9}$ This prevents nanobubbles from rising to the free surface where the change in pressure of the atmosphere can cause a shift in the equilibrium between the pressure on the inside of the bubble and the pressure on the outside of the bubble, thereby causing the bubble to become unstable and burst. ${ }^{9,11,14,15}$

Other explanations involve the physical chemistry of the nanobubbles. The electrically charged interface of the bubble creates strong repulsive forces between bubbles, which prevent bubble coalescence, bubble bursting, and Ostwald ripening. Others speculate that the bulk nanobubble stability is a result of the presence of "universal" contaminants. ${ }^{9-11,14}$ The contaminants are organic or surfactant molecules that form a shell around the nanobubbles, that reduces interfacial tension, thereby decreasing the Laplace pressure within the bubble, and preventing dissolution. ${ }^{\mathbf{1 4 , 1 6 - 2 0}}$ Despite all of these explanations, to date, the literature has not converged on a single theory that explains the apparent stability of bulk nanobubbles, but bulk nanobubble technology has nevertheless continued to progress, leading to new applications. Applications of bulk nanobubbles are in the food, water and cleaning industries., ${ }^{\text {,21-24 }}$ Bulk nanobubbles are also useful in several biomedical applications. The benefits of nanobubbles in biomedical applications are related to their biocompatibility, customizability, stability, and small size.

Several production techniques have been employed to produce bulk nanobubbles using mechanical and chemical production methods. However, many of these methods generate highly polydisperse bubble populations and lack controllability over size and concentration. Furthermore, some of these production systems use reagents inefficiently, leading to excessive waste of costly experimental materials. In this review, we suggest the utilization of microfluidics and propose possible microfluidic implementation methods to address some of the current challenges in making bulk nanobubbles.

\section{Biomedical applications}

\subsection{Biomedical imaging}

2.1.1 Ultrasound contrast enhancement. One of the most reported biomedical applications of bulk nanobubbles is ultrasound image contrast enhancement. Gas bubbles within tissues can act as harmonic oscillators and oscillate/resonate in response to ultrasound excitation due to the compressibility of the encapsulated gas. ${ }^{25}$ The second harmonic increases the intensity of backscattered ultrasound signals, which results in greater signal intensity in tissues with bubbles present than in background tissue. ${ }^{\mathbf{2 6 - 3 0}}$ Researchers and clinicians have been using microbubbles to achieve this ultrasound intensity enhancement effect in blood pool imaging for decades; however, nano-scale contrast agents offer unique benefits which cannot be achieved using micro-scale agents. ${ }^{31-34}$ Namely, nanoscale agents are small enough to extravasate from the leaky tumour vasculature as a result of the improved permeability and retention effect (EPR). ${ }^{35,36}$ 
Extravasation via the EPR effect is unique to nano-scale agents. Extravasation of the contrast agent affords clinicians insight into the tissue of interest and allows functionalized bubbles to target surface markers of tumour cells. ${ }^{36,37}$ There is also evidence that nanobubbles produce a similar quality ultrasound image compared to microbubbles despite the lower backscatter intensity per bubble. ${ }^{38,39}$ Perera et al. have conducted research to improve the stability and extravasation ability of bulk nanobubbles for ultrasound contrast enhancement. ${ }^{40}$ In 2010, they showed that incorporating Pluronic surfactants into the lipid shell before adding the gas core was effective at yielding sub-micron bubbles without reducing echogenicity or stability. ${ }^{32}$ In more recent work, they demonstrate that incorporating crosslinked $N, N$-diethylacrylamide and $N, N$-bis(acryoyl) cystamine networks into the lipid shell of the Pluronic nanobubbles increases ultrasound signal intensity and reduces the decay rate of nanobubbles both in vitro and in vivo. ${ }^{\mathbf{4 0}}$

2.1.2 Photoacoustic contrast enhancement. Photoacoustic imaging can also benefit from the unique physical characteristics of nanobubbles. Photoacoustic imaging techniques use the photoacoustic effect to generate an image of the target tissue. To use this effect clinically, short wave light pulses are used to excite chromophores, optically absorptive molecules in tissue which create tissue thermoelastic expansion. The resulting pressure wave is sensed by an ultrasound transducer, which obtains the spatial distribution of the chromophores..$^{\mathbf{3 9 4 1 - 4 4}}$

Chromophores can be encapsulated within microbubbles and nanobubbles, which increases their circulation half-life, and reduces the molecular interactions between chromophores and proteins in the bloodstream that may cause inconsistent spectral characteristics. ${ }^{42}$ Relatively little work has been published about nanobubbles in photoacoustic imaging. However, Kim et al. report poly (lactic-co-glycolic acid) (PLGA) shell nanobubbles effectively encapsulating India ink and providing photoacoustic contrast enhancement in vitro, and Bodera et al. report lipid shell nanobubbles stained with Sudan black producing strong photoacoustic signals in vitro. ${ }^{39,40,42-46}$

2.1.3 Molecular imaging. In molecular imaging, functionalized nanobubbles may also be used in conjunction with ultrasound or photoacoustic imaging modalities. Nanobubbles may be functionalized with molecules that have an affinity for markers on tissues or cells of interest. Ultrasound or photoacoustic imaging is then used for spatiotemporal tracking of the nanobubbles in vivo using their contrast-enhancing properties, which reveal the activity of the target tissues or cells. ${ }^{47-49}$

This technique can be used to track the molecular changes that accompany oncogenesis and metastasis and predict the optimal therapeutic strategy for different types of tumours. ${ }^{\mathbf{5 0 , 5 1}}$ Yang et al. use phospholipid-shell-perfluoropropane-core nanobubbles conjugated with biotinylated anti-ErbB2 affibody molecules to induce affinity to HER2-overexpressing tumour targeting (HER2 overexpressed in breast, ovarian, and urinary bladder carcinomas). ${ }^{36}$ The affibody-functionalized nanobubbles exhibit significantly greater signal intensity than commercial Sonovue ${ }^{\mathrm{TM}}$ and unconjugated nanobubbles. Perera et al. report prostate-specific membrane antigen (PSMA)targeted nanobubbles increasing accumulation and retention in PC3-pip tumours compared to non-functionalized nanobubbles and commercial Lumason ${ }^{\mathrm{TM}}$ microbubbles. $^{51}$

Wu et al. used anti-CD25 antibody functionalized nanobubbles and ultrasound to diagnose acute rejection after cardiac transplantation (Fig. 2). ${ }^{47}$ The functionalized nanobubbles are bounded to T-cells in the myocardium after extravasation through the vessel wall. Rejection is characterized by a temporal delay in the ultrasound contrast enhancement and prolonged enhancement compared to nonspecific (non-functionalized) nanobubbles. The authors hypothesize that the second ultrasound intensity peak, observed in the allografts but not the isografts, results from an accumulation of functionalized nanobubbles bound to T-cells executing an immune response in the myocardial interstitium.

\subsection{Dentistry}

In dentistry, regeneration from stem cells is used to treat diseases and disorders related to teeth. In vitro culturing of human dental follicle stem cells (DFSCs) is one of the branches of dentistry that may benefit from bulk nanobubbles. In the research by Oishi et al., air and oxygen nanobubbles are used to increase the amount of in vitro-produced DFSCs. ${ }^{52}$ The presence of air/oxygen nanobubbles in the culture media of DFSCs improve the proliferation of DFCSs; while the reason for this phenomenon is still not clear, the air/ oxygen nanobubbles may alter the growth behaviour of the cells. ${ }^{52}$

Another application of bulk nanobubbles in dentistry is in the treatment of periodontal diseases. Arakawa et al. use a solution of water and ozone nanobubbles to treat patients suffering from bone loss and inflammation due to peri-implantitis. ${ }^{53}$ Irrigation of the affected area with ozone nanobubbles for 12 weeks profoundly reduced the number of bacteria present, resulting in the elimination of inflammation and recovery of the damaged bone. They show that chemical plaque control with ozone nanobubble therapy, used in conjunction with mechanical plaque control via sonic toothbrush, is sufficient to eradicate the peri-implantitis, eliminating the need for surgical intervention..$^{53}$ This treatment is also available using ozonated water, but the 30 minute half-life of ozonated water is prohibitively short for home use by the patient.

The ozone nanobubble solution is stable for 6 months, which allows the patient to complete the therapy without having to visit the clinic daily. The antimicrobial properties of ozone can also be used to enhance conventional periodontitis treatment consisting of subgingival debridement. ${ }^{54}$ Other antimicrobial agents such as chlorhexidine and hydrogen peroxide are often used with debridement to reduce the number of subgingival pathogens in periodontitis treatment. Still, these agents have some drawbacks, which include causing mucosal diseases, altering the taste sensation, and staining of teeth. ${ }^{54}$ To improve on the deficiencies mentioned above, Hayakumo et al. proposed ozone nanobubbles as a biocompatible adjunct to periodontal therapy. By carrying out clinical tests on patients with periodontal disease, they achieve a decrease in the probing pocket depth and subgingival plaque bacteria without any serious side effects. ${ }^{54}$

\subsection{Oxygen delivery}

2.3.1 Wound healing. Hypoxia and ischemia-reperfusion are the primary reasons for insufficient oxygen levels during 

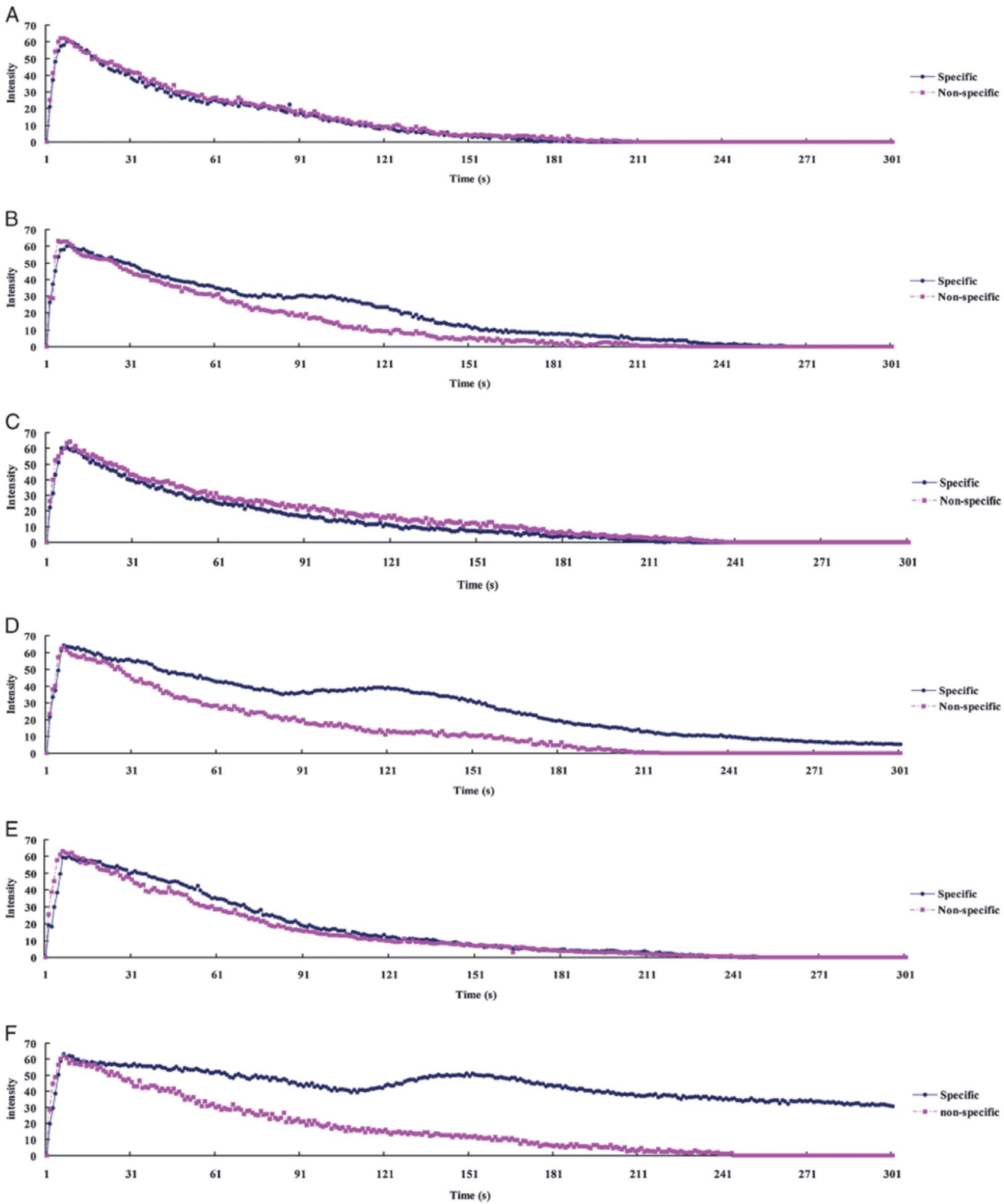

Fig. 2 Ultrasound time-intensity curves (TIC) from myocardial contrast echocardiography of allografts and isografts imaged with functionalized and non-functionalized nanobubbles. ( $A, C$, and E) show TIC for isografts at 2, 4, and 6 days post transplantation, respectively. (B, D, and F) show TIC for allografts at 2, 4, and 6 days post transplantation, respectively. Note the delayed intensity peak in for allografts imaged with functionalized nanobubbles. ${ }^{47}$

the wound healing process. Lack of enough oxygen in a wounded tissue extends the healing period, leading to irreparable damages, particularly in patients with diabetes. ${ }^{55-57}$ Two of the conventional treatments for maintaining the desired oxygen level in a wound are hyperbaric oxygen and trans-obturator tape therapy. ${ }^{58-61}$ However, these treatments are costly and 
often cause cellular toxicity. Moreover, they are not useful for all kinds of wounds. A replacement for the traditional therapies is utilizing oxygen nanobubbles, as their stability in solution helps increase the oxygen level in a wound over a prolonged period. Another advantage is their ability to trap a volume of oxygen, which can provide sufficient oxygen for the treatment by injecting them into the damaged tissue. In addition to the oxygen delivery benefits, the negative surface charge of oxygen nanobubbles attracts debris and enhances the cleaning of the wound. ${ }^{62}$

2.3.2 Tumour hypoxia treatment. Tumour hypoxia may cause the survival of cancer cells in hypoxic areas. ${ }^{63,64}$ Furthermore, in standard cancer therapies such as radiation and photodynamic therapy in which obtaining the desired result depends on the sufficient level of oxygen in the tumour, hypoxia deteriorates the effectiveness of the treatment. ${ }^{63-66}$

One of the possible methods to supply oxygen to tumour cells is using lipid-coated oxygen nanobubbles. The lipids on the shell of the nanobubbles increase their stability, preventing the release of oxygen before reaching the tumour. When using nanobubbles, the release of the oxygen cargo is controllable and can be performed by ultrasound stimulation. ${ }^{67,68}$ In a novel method proposed by Song et al., the nanobubbles are coated with a shell sensitive to $\mathrm{pH}$ changes. ${ }^{69}$ Acetylated dextran is used as the $\mathrm{pH}$-change sensitive polymer shell, which is disrupted in the acidic environment of tumour cells ( $\mathrm{pH}$ 6-6.5) and releases oxygen within the tumour. ${ }^{69}$

\subsection{Tissue or organ-selective gene delivery by the combination of ultrasound and nano/microbubbles or bubble liposomes}

Viruses have evolved to be efficient delivery systems for nucleic acids to specific cell types while avoiding immunosurveillance. This makes viruses an attractive delivery vehicle for gene delivery. In gene therapy, these types of viruses are also known as viral vectors. ${ }^{71}$ There are several types of laboratory viruses that have been modified to suit specific applications; however, their use has significant limitations. Retroviral vectors allow for stable integration within the host cell; however, transduction requires mitosis to occur, which can contribute to genomic instability leading to cancer. ${ }^{71}$ For transient gene expression, adenoviral vectors are desirable due to their efficient transduction of genes; however, for genetic disorders where stable long-term gene expression is needed, adenoviral vectors would not be ideal. Herpes simplex virus (HSV) vectors are best known for their ability to infect nondividing cells and their delivery of exogenous DNA; however, cell damage can occur due to their cytotoxic risks. Adeno-associated virus (AAV) vectors pose the lowest immunogenic risk among all of these viruses; however, AAV vectors have small cloning capacities, limiting their ability to transduce larger genes greater than $5 \mathrm{~kb}^{71}$

In recent years, ultrasound-mediated gene delivery systems with nanobubbles have been developed as a non-viral vector gene delivery system that helps overcome some shortfalls of conventional viral approaches. ${ }^{70,72,73}$ In this system, transient pores are opened, by utilizing nano and microbubbles that interact with ultrasound, to disrupt the cell membrane. As a result, these pores allow the transfer and transduction of genetic material into the host cell without the need for endocytosis (Fig. 3). This method is advantageous as it provides a non-invasive and tissue-specific gene expression approach to manipulating and exposing targeted tissues and organs with therapeutic genes through the use of ultrasound. Further, the use of lipid-coated nanobubbles also provides low cytotoxic effects in vivo, reducing the inherent risk of vector-specific immune responses and toxicity that is associated with using pathogenic viruses. ${ }^{70}$

Kida et al. examined the effectiveness of nanobubbles for gene delivery using a commercially available handheld ultrasound scanner. Two studies were completed, one in vitro and one in vivo, with controls taken for each experiment. ${ }^{73}$ In the in vitro experiment, luciferase-expressing pDNA is introduced into HSC2 cells. After 24 hours, they found an increase in the luciferase expression proportional to the concentration of the nanobubble in the solution. A similar effect was also observed when the experiment was completed in vivo with ddY mice. In both groups, a significant increase in expression of the luciferase with the use of nanobubbles and ultrasound was observed. ${ }^{73}$

2.4.1 Plasmid DNA delivery. In vitro studies have previously been performed using the transfection method where cells suspended with microbubbles and plasmid DNA are exposed to ultrasound for up to tens of seconds. Here, transfection efficiency is affected by ultrasound exposure conditions, such as intensity, frequency, period, duty cycle, type, and microbubble concentration. However, optimized conditions are not well understood to maximize transfection efficiency. Aoi et al. developed herpes simplex virus mediated thymidine kinase (HSV-tk)-mediated
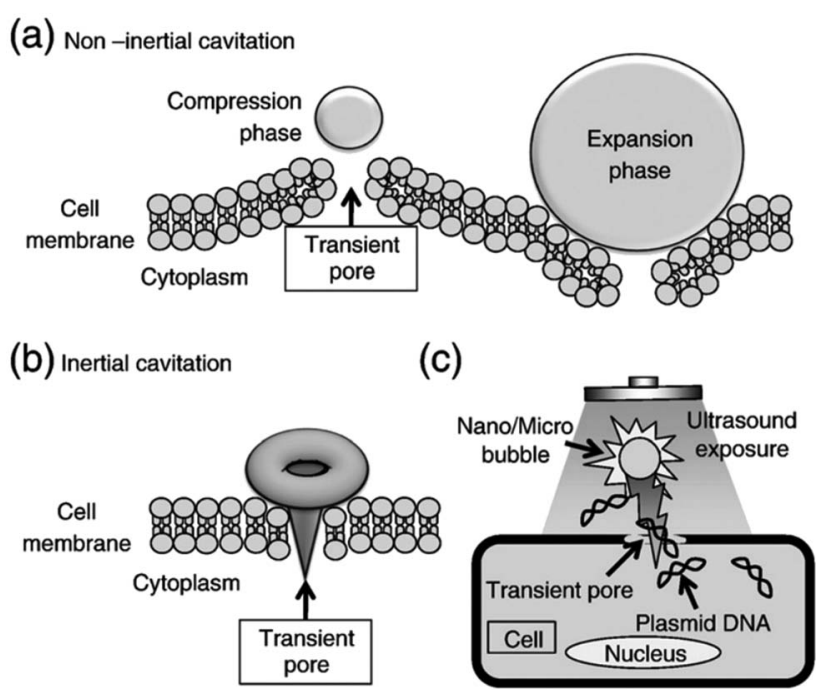

Fig. 3 Schematic diagram depicting the pore formation and delivery of fluids and macromolecules in the cell membrane. (a) Non-inertial cavitation causing pushing and pulling behaviour along the cell membrane due to the expansion and compression of bubbles. (b) Inertial cavitation collapsing the bubble, rupturing the cell membrane and creating a transient pore. (c) Transmembrane fluid and macromolecules, including plasmid DNA and oligonucleotides, transported by nano/microbubbles travelling into cells through a transient pore. ${ }^{70}$ 


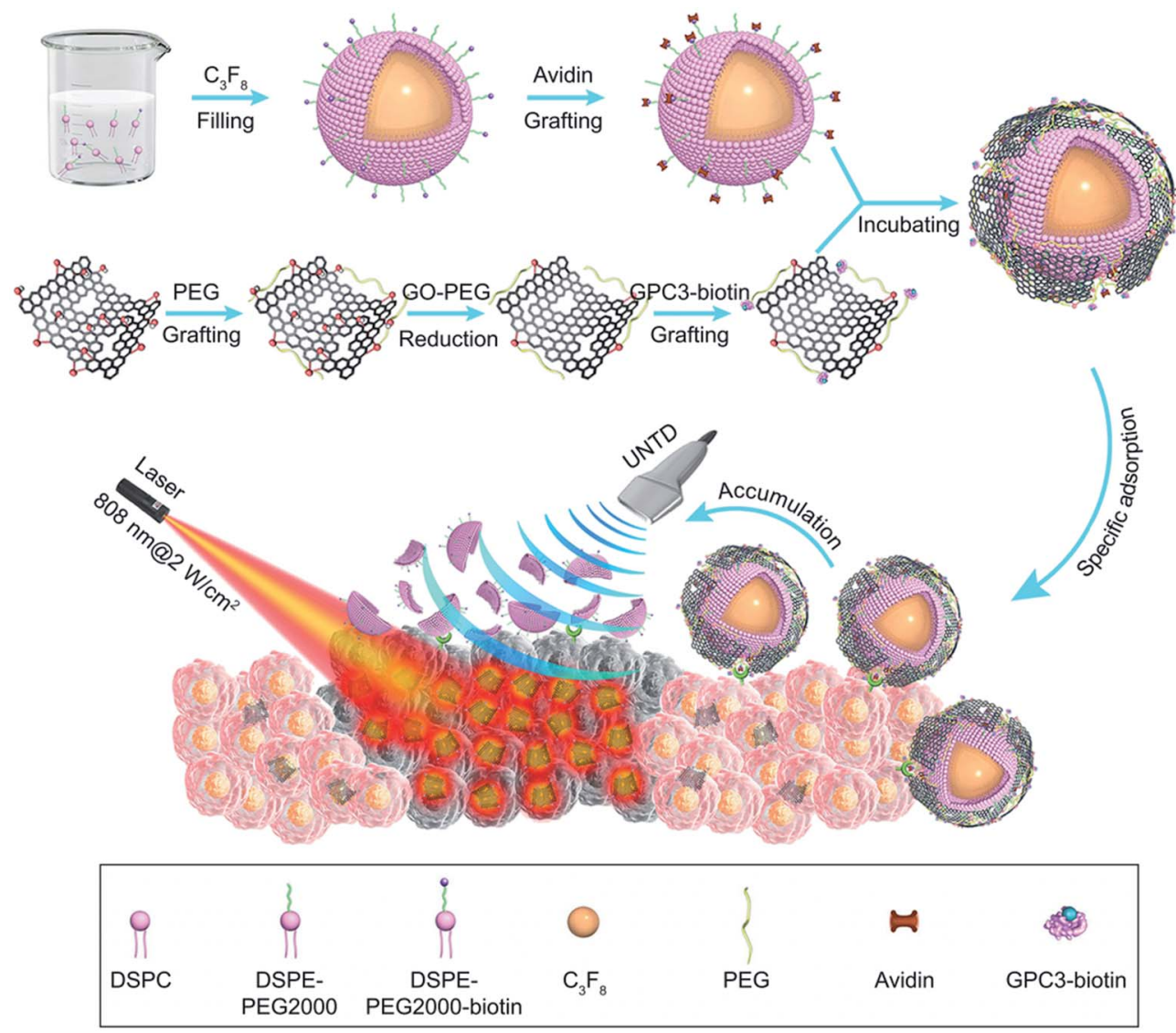

Fig. 4 Preparation and use of nanobubble-GP3-rGO functionalized nanobubbles for NIR photothermal ablation of $\mathrm{HCC}^{76}$

suicide gene treatment utilizing commercial Optison ${ }^{\mathrm{TM}}$ nanobubbles and ultrasound. ${ }^{74}$ In this therapy, HSV-tk corded plasmid DNA and nanobubbles are injected into tumour tissue of mice, and ultrasound is transdermally exposed toward the targeted tissue. The reduction of tumour size is observed by administration of ganciclovir in the mice transfected HSV-tk corded plasmid DNA with nanobubbles and ultrasound. ${ }^{74}$

2.4.2 Oligonucleotide delivery. Oligonucleotides are short single- or double-stranded polymers of nucleic acid that can stop the expression of a specific gene and be used to potentially develop new disease treatments for malignant, infectious, and autoimmune diseases. ${ }^{70}$ To achieve effective gene silencing, common oligonucleotides such as antisense, decoy and siRNA must be delivered to the cytoplasm of targeted cells to function properly. Combining ultrasound and nanobubbles has been found as a useful method for delivering extracellular molecules into the cytosol where oligonucleotides function best ${ }^{70}$ In an experiment conducted by Negishi et al., they reported that siRNA is directly introduced into the cytoplasm with only 10 seconds of nanobubble and ultrasound exposure, providing an effective and efficient transfection of the oligonucleotide. ${ }^{75}$

\subsection{Tumour ablation}

Thermal ablation is the process of inducing coagulative necrosis in target tissues by producing transient temperature increase using high intensity focused ultrasound (HIFU), radiofrequency (RF) irradiation, microwave irradiation, laser irradiation, cryoablation, and irreversible electroporation. ${ }^{77}$

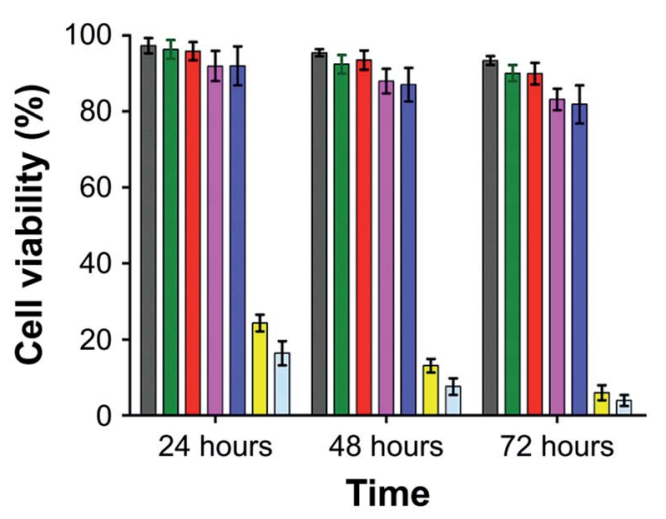

$\square$ Control $\square$ UTND $\square$ Laser
$\square$ NBs-GPC3-rGO
$\square$ NBs-GPC3-rGO + UTND
$\square$ NBs-GPC3-rGO + laser
$\square$ NBs-GPC3-rGO + UTND + laser

Fig. 5 Comparison of tumour cell viability from different treatment methods tested. The combined therapy with NIR laser + ultrasound + functionalized nanobubbles yielded the best results, with a 72 hour cell viability of $2.26 \%{ }^{76}$ 
These techniques are often applied to non-invasively manage unresectable tumours of the liver, pancreas, bone, kidney, and lung. ${ }^{77-80}$ Several studies have shown that bulk nanobubbles can directly increase the effectiveness of HIFU by increasing acoustic energy deposition in target tissues due to shear stress, cavitation, and streaming induced by nanobubble oscillation and collapse, and by controlled release of thermal sensitizers and anti-cancer drugs to the tumour. ${ }^{81-83}$ Yao et al. show that lipid-shell nanobubbles and Sonovue ${ }^{\mathrm{TM}}$ Microbubbles exhibit similar volumes of coagulative necrosis, compared to nanobubbles, in in vivo rabbit breast tumour models and excised bovine livers for identical HIFU conditions, indicating that nonfunctionalized nanobubbles are an effective enhancer of HIFU tumour ablation. ${ }^{84}$

In another study, Liu et al. use target nanobubbles and ultrasound to visualize and deliver reduced graphene oxide (rGO) nanosheets to in vitro hepatocellular carcinoma (HCC) HepG2 cells for enhanced near-infrared photothermal ablation. ${ }^{76}$ They conjugate glypican-3 (GP3), as a targeting molecule for HepG2 cells, and rGO, for its photo-absorbing properties, to avidinylated phospholipid-shell nanobubbles to both deliver the rGO to HCC HepG2 cells and provide ultrasound contrast enhancement to visualize the ablation process (Fig. 4).

The combined NIR laser, ultrasound, and functionalized nanobubbles therapy result in a 72 hour HCC HepG2 cell viability of $2.26 \%$, significantly lower than the other treatment methods tested, shown in Fig. 5. These studies have demonstrated the ability of bulk nanobubbles to provide synergistic effects when paired with HIFU, laser and RF ablation. Compared to microbubbles, which have also been used for these types of treatments, tumour ablation therapies can benefit from the increased blood circulation lifetime and tumour targeting ability offered by bulk nanobubbles as a result of the EPR effect. ${ }^{76}$ Table 1 summarizes the biomedical applications of bulk nanobubbles.

\section{Characterization}

Before the utilization of bulk nanobubbles in various applications, it is often necessary to first investigate the nanobubbles' characteristics, including concentration, size, and

Table 1 Summary of the biomedical applications of bulk nanobubbles

\begin{tabular}{lll}
\hline Current biomedical applications & Summary & Reference
\end{tabular}

Ultrasound contrast enhancement

Molecular imaging

Dentistry

Wound healing

Tumour hypoxia treatment

Non-viral vector gene delivery

Tumour ablation
Gas bubbles act as harmonic oscillators, taking advantage of the compressibility of the encapsulated gas to resonate with ultrasound frequencies and increase the intensity of the backscattered signal. This produces sonograms with increased contrast due to the high difference in echogenicity

Nanobubbles have shown to be effective mediums for photoacoustic contrast enhancement. Nanobubble shells can be functionalized with antigens, antibodies and other high biomarking affinitive molecules to increase the specificity of photoacoustic imaging

Nanobubbles can be an ideal medium as molecular imaging markers in conjunction with ultrasound or photoacoustic imaging modalities to track molecular changes and predict optimal therapeutic strategies for different types of tumours. Nanobubbles can be functionalized with molecules that target biomarkers of specific tissues and cells of interest. The acoustic properties of the bubbles can be used to temporally and spatially track the bubbles to understand tissues on a molecular level

Bulk nanobubbles are utilized to stimulate the growth of dental follicle stem cells and improve proliferation. Bulk nanobubbles have been employed as dental bone reconstruction therapy and for their anti-periodontopathic bacterial properties

As a replacement for hyperbaric oxygen and trans-obturator tape therapy, oxygen nanobubbles can be injected into damaged hypoxic tissue and deliver oxygen. Further, the negative charge of the oxygen nanobubbles aid in the debridement process

Hypoxic environments deteriorate tumour treatment therapies and promote the survival of cancer cells. Lipid-coated nanobubbles have been applied to deliver oxygen to tumours to allow for more effective treatment Ultrasound-mediated gene delivery systems make use of nanobubbles to induce micro streams and microjets to create transient pores which allow for genes to be delivered into cells

High intensity focused ultrasound and radiofrequency irradiation for tumour ablation benefit from the synergistic effects of bulk nanobubbles. They enhance the effectiveness of these treatments by increasing the acoustic energy deposition in target tissues through bubble oscillations and collapse as well as by the controlled release of thermal sensitizers and drugs to tumours
25, 29-37 and 85-87

$38-40,42$ and 44

$43,45,46,48$ and 49

47 and $50-54$

55-61 and 88

$63-69$

$70,71,73-75$ and $89-91$

83 and 84 
polydispersity index, representing the nonuniformity of a sample based on size. Due to the small scale, nanobubble measurements are difficult. In this section, we discuss current characterization methods for bulk nanobubbles.

\subsection{Dynamic light scattering (DLS)}

DLS is used to describe a broad set of techniques for characterizing particle suspensions in liquids. These techniques typically involve measuring the diffusion of particles in a suspension and calculating the particle size using a theoretical relationship. A monochromatic light source is directed through the sample, and the particles of interest scatter the light in all directions. The dispersed light is received (typically by a photomultiplier) and produces a signal. The scattered light undergoes constructive and destructive interference at different locations, resulting in spatially varying signal intensity which is tracked over time. ${ }^{92,93}$ As the suspended particles undergo Brownian motion, the scattered light intensity fluctuates over time, and the spatial and time fluctuations of the signal are detected by an autocorrelator to measure the time scale of particle motion. The autocorrelation data is then used to calculate the size distribution of the suspended particles using models which account for the temperature and solvent viscosity. ${ }^{\mathbf{9 4 , 9 5}}$

DLS techniques allow for the sample particles to remain in the liquid during characterization. Keeping particles in suspension during characterization ensures that bulk properties, such as concentration and size distribution, can be accurately measured, and that individual particles are not modified by the drying or evacuation processes necessary for some other size characterization techniques including electron microscopy. Though DLS is widely used to characterize nanobubble suspensions, it is not an ideal measurement technique for lipidshelled nanobubbles or suspensions in which other nanoparticles may be present because it cannot distinguish between gas bubbles and other solid particles. ${ }^{\mathbf{9 6 , 9 7}}$

\subsection{Resonant mass measurement (RMM)}

This method was first introduced by T. P. Burg et al. in $2007 .{ }^{98}$ The technology contains a silicone microcantilever continually oscillating at its resonance frequency. A microchannel is embedded inside the microcantilever, and a fluid containing nanoparticles passes through the microchannel (Fig. 6).$^{98}$ When a nanobubble containing fluid passes through the microchannel, the resonance frequency changes. An electric circuit that continually monitors the frequency of microcantilever converts the shift in frequency to the buoyant mass of the nanobubbles. ${ }^{98}$

One of the advantages of this technique is that nanobubbles could be easily distinguished from non-gaseous nanoparticles. When the floating particle in the channels is a nanobubble, the resonance frequency increases. In contrast, for the nanoparticles other than nanobubbles, it decreases. For size measurements, the density of the particle needs to be known. One disadvantage of the RMM method is the high probability of microchannel blockage. Therefore, samples should be refined via a filter with pores smaller than the microchannel width and height before any tests.

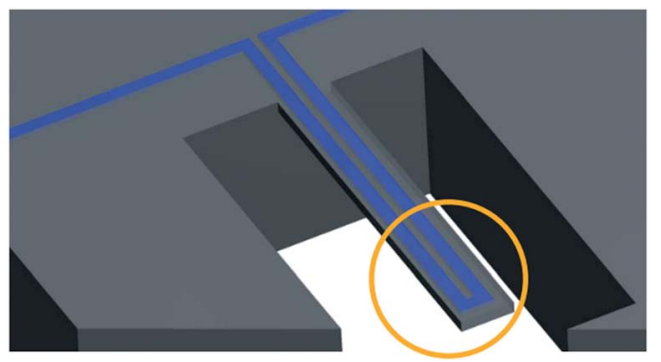

Fig. 6 An embedded microchannel in a silicon microcantilever. The circled section highlights the tip of the microcantilever where the sensitivity is maximum. ${ }^{98}$

\subsection{Nanoparticle tracking analysis (NTA)}

Nanoparticle tracking is a system for sizing particles from around 30-1000 nm with the lower limit of detection being dependent on the refractive index of the nanoparticles. NTA utilizes video sequences to analyse Brownian motion through the illumination of the particles in a sample with a laser beam. The scattered light from particles is detected with a charged coupled device (CCD) or complementary metal oxide semiconductor (CMOS) camera and converted to a digital signal for recording. Specialized algorithms are used to detect individual particles and track their path (Fig. 7). ${ }^{99}$

NTA has the capability of sample visualization and can provide an approximate particle count and concentration. Mean size values that are obtained by NTA are smaller and closer to the expected results of particle size tests compared to DLS. ${ }^{100}$ Further, NTA can profile the size distribution of particles to display the number of particles. As well, NTA allows for clear distinction between different sizing populations and the ability to search and scan the sample for desired particles with the system's ability to separately track every visible particle. One of the main drawbacks with NTA is the challenge of the operator to identify and set optimal sample compositions and instrument settings such as narrow particle concentration, and particle properties (shape,

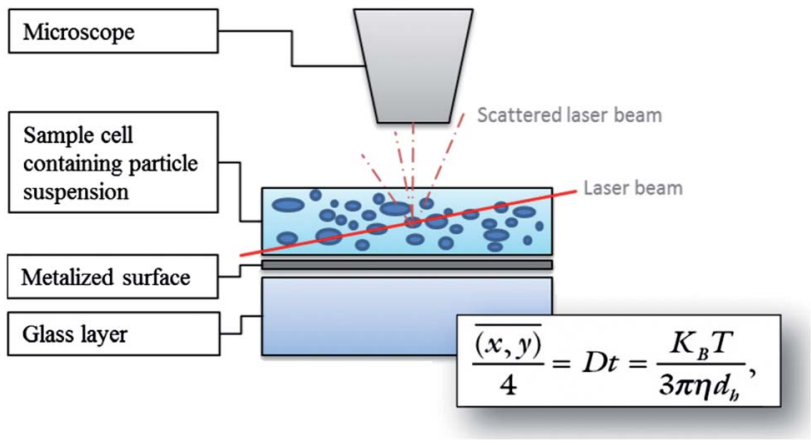

Fig. 7 Diagram illustrating the principle of NTA measurements using the Stokes-Einstein equation. Particles are illuminated by a laser light and movements of the particles are recorded through the scattered light via a CCD by a microscope. The software tracks the Brownian motion of each particle by determining the diffusion coefficient, and then calculates the size as the mean square of the particle path using the Stokes-Einstein equation. ${ }^{99}$ 
refractive index and background noise) in order to yield the most accurate results. Moreover, NTA requires particle concentration between $10^{7}$ to $10^{9} \mathrm{~mL}^{-1}$ while the required sample concentration for the DLS technique is less critical. ${ }^{101-104}$

\subsection{Electron microscopy}

Two less common techniques to characterize bulk nanobubbles are scanning electron microscopy (SEM) and transmission electron microscopy (TEM). These methods are commonly used to image the structure and the presence of bulk nanobubbles in a sample. ${ }^{\mathbf{1 0 5 , 1 0 6}}$ Since they can only provide small fields of view, they are not suitable for determining the concentration and average size of bulk nanobubbles in a sample. In electron microscopy, a beam of high voltage electrons is generated and accelerated in a chamber composed of different apertures and electromagnetic lenses towards the sample. In SEM mode, the reflected electrons from the sample are collected and analysed to achieve the sample surface's image. While in TEM mode, the transmitted electrons are received and interpreted to collect information about the sample's inner structure, including the crystal structure and morphology. TEM has better resolution $(<50 \mathrm{pm})$ in comparison to SEM $(\approx 0.5$ $\mathrm{nm}$ ); however, it is a more costly method than SEM. Furthermore, TEM's field of view is considerably smaller than SEM and can image a minimal area of the sample. ${ }^{105-108}$

\subsection{Cryo-EM visualization of nanobubbles}

The sample containing the nanobubble suspension may be rapidly frozen prior to electron microscopy to use a technique known as cryogenic electron microscopy (cryo-EM). Using a cryogen such as liquid nitrogen to freeze the sample quickly causes the nanobubbles to be trapped in the resulting amorphous ice, which maintains the sample's morphology without deviations introduced by the crystallization of water. ${ }^{109}$ The trapped bubbles can then be imaged directly with TEM while keeping the sample frozen as in reports of Li et al. ${ }^{109}$ and Hernandez et al. ${ }^{\mathbf{1 1 0}}$ Alternatively, the frozen sample can be fractured, and the voids in the fracture planes representing the shells of the frozen bubbles can be coated for subsequent SEM or cast for subsequent SEM or TEM characterization. ${ }^{109-111}$

\section{Current nanobubble manufacturing methods}

The performance of micro- and nanobubbles in the biomedical applications, and therefore treatment outcomes, are related to the bubble manufacturing methods currently employed. In the paragraphs below, we outline the methods currently used to create nanobubble mixtures.

\subsection{Mechanical methods}

4.1.1 Agitation. For the purposes of this paper, "agitation" refers to mechanical shaking of a sealed vessel in which there exists a gas, destined to be the bubble core, and a liquid, containing bubble shell materials, in contact with each other at an interface. Agitation of this vessel introduces gas into the liquid
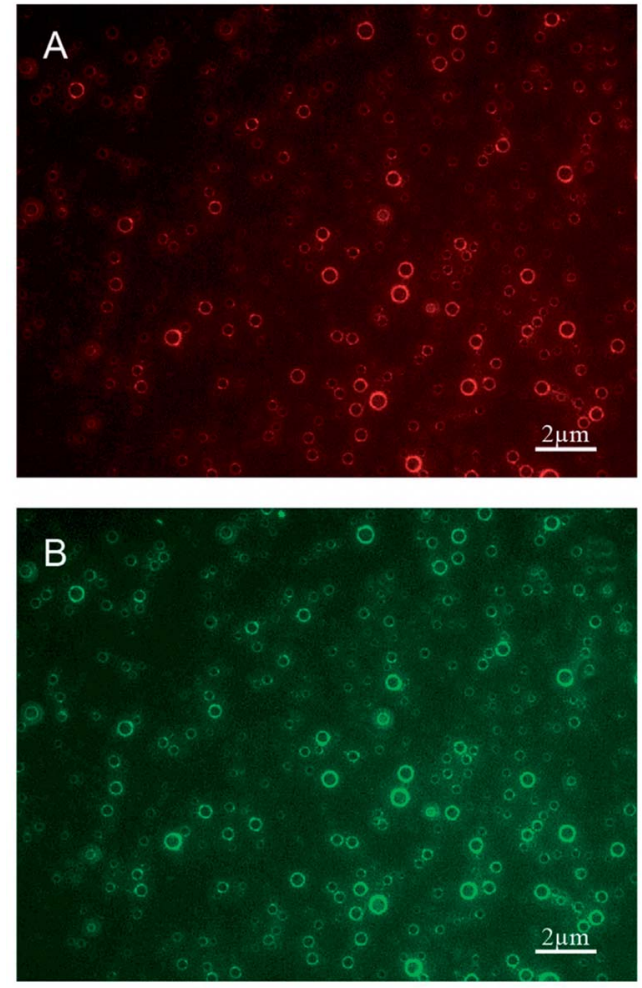

Fig. 8 Polydisperse nanobubbles functionalized with Cy3-labeled cell-penetrating peptide (CPP) and FITC-labeled epidermal growth factor receptor-targeted small interfering RNA (siEGFR), fabricated using the lipid film hydration agitation method for gene therapy of triple negative breast cancer. (A) Nanobubbles fluorescing red, indicating effective loading of the CPPs in the bubble shell. (B) The same nanobubbles fluorescing green, indicating effective loading of the siEGFR in the bubble shell. ${ }^{112}$

to form bubbles. One of the early agitation methods to fabricate lipid-shell nanobubbles, described by Krupka et al., involves dissolution of a solid lipid mixture in chloroform, followed by evaporation of the chloroform..$^{32}$ Liposomes are then formed by hydration of the lipid film with a PBS-glycerol solution in an incubator-shaker. The resulting solution is placed in vials which are sealed with septum caps, and the air is removed and replaced with octafluoropropane. The vial is then shaken, or agitated, in a VialMix ${ }^{\mathrm{TM}}$ shaker for $45 \mathrm{~s}$ to form a polydisperse suspension of micro- and nanobubbles. The suspension is then centrifuged with the vial inverted to isolate the nanobubbles from the microbubbles, after which the nanobubbles can be removed from the suspension by withdrawing the bottom $5 \mathrm{~mm}$ of solution with a $21 \mathrm{G}$ needle. ${ }^{32}$

Many extensions of the aforementioned method involve the same mechanism of bubble formation, but incorporate other molecules in the lipid shell to enhance stability, echogenicity, size, and surface affinity. One such method, described by the same group, requires dissolution of the solid lipid mixture in a PBS-propylene glycol solution at elevated temperatures, followed by addition of glycerol. ${ }^{\mathbf{1 1 3}}$ This solution is then transferred to sealed vials and agitated as described earlier. The inclusion of glycerol in the bubble shell increases the stiffness 
of the shell as a result of hydrogen bonding, while the propylene glycol increases the flexibility of the shell. ${ }^{113}$ With both glycerol and propylene glycol included in the shell, the bubbles exhibited superior in vivo stability compared to both FDA-approved Lumason $^{\mathrm{TM}}$ ultrasound contrast agent, and Pluronic nanobubbles.

Other extensions of the agitation method can be found in the literature. ${ }^{2,112,114}$ Fig. 8 shows functionalized bulk nanobubbles with RNA. ${ }^{112}$ Tian et al. report a novel method for producing bulk nanobubbles using a gas-liquid mixing pump to introduce free nanobubbles in a degassed aqueous solution. ${ }^{115}$ This method is similar to the agitation methods described earlier in that it involves the production of a lipid film via dissolution and subsequent solvent evaporation. A gas-liquid mixing pump is used to mix degassed and deionized water (DI water) with sulfur hexafluoride to create an aqueous suspension of nanobubbles with a sulfur hexafluoride core. This suspension is then added to a vessel containing the lipid film and allowed to incubate for hours to ensure self-assembly of the lipid molecules at all gasliquid interfaces. This method does use mechanical agitation in the gas-liquid pump as the primary mechanism of creating bubbles, but self-assembly of the lipid molecules occurs after agitation, as opposed to the simultaneous bubble formation and self-assembly in other methods. ${ }^{112}$

Agitation can yield very high bubble concentrations, $10^{11}$ $\mathrm{mL}^{-1}$, affording the user a significant range of possible concentrations via post-fabrication dilution as required. ${ }^{97}$ This flexibility allows researchers to tune the bubble suspension to suit the application; for example, to avoid acoustic shadowing in ultrasound contrast enhancement, the nanobubble suspension should be diluted to $10^{8}$ to $10^{9} \mathrm{~mL}^{-1} \cdot{ }^{97}$ As with many fabrication methods, it is not possible to control the monodispersity of the bubble population in real time. Consequently, researchers typically centrifuge the bubble suspension and remove a small volume from the bottom to isolate nanobubbles from microbubbles, yielding a sample of polydisperse nanobubbles while the remainder of the suspension is discarded as waste. ${ }^{116}$

4.1.2 Double-emulsion solvent evaporation. Kim et al. describe a process for making nanobubbles in which poly (lactic-co-glycolic acid) (PGLA) and methylene chloride solution, polyvinyl alcohol (PVA) and India Ink solution, and PVA solution form a double-emulsion via ultrasonication. ${ }^{39}$ The double emulsion is added to an isopropanol solution and stirred. The middle phase of the double emulsion is polymerized to create spherical shells, which are used as nanobubble shells following a process of washing, centrifugation, and freeze-drying. The India Ink is loaded in the bubble shell prior to polymerization for use as a chromophore for photoacoustic imaging. Several other groups have created polymer shell nanobubbles using similar protocols, and have conjugated the nanobubbles with molecules such as tumour-targeting proteins and anticancer drugs. ${ }^{\mathbf{8 3 , 1 1 7}}$

The primary benefit of this technique is that the bubbles can be fabricated in large quantities due to the bulk nature of the ultrasonication-based emulsification process. Conversely, it suffers from a lack of real-time control over the size and monodispersity of the emulsion, both of which are important properties to maximize bubble ultrasound excitation resonance in contrast enhancement or drug delivery applications.

\subsubsection{Hydrodynamic cavitation}

4.1.3.1 Hydrodynamic cavitation by a baffled high intensity cell. In this method, bulk nanobubbles are produced using an apparatus called "baffled high-intensity agitation (BHIA) cell". This setup consists of a shaft that is connected to a high-speed agitator, two valves to take samples for characterization, and thumbscrews to highly seal the lid of the cell to the body. As Fig. 9 shows, a solution fills the cell, and an impeller is placed such that it does not make contact with any part of the cell. The impeller is then rotated at different RPM to create nanobubbles by hydrodynamic cavitation. The solution used in this technique is a mixture of Milli-Q water, $\mathrm{KCl}$ as the electrolyte, $\mathrm{NaOH}$ and $\mathrm{HCl}$ as $\mathrm{pH}$ modifiers, and sodium dodecyl sulphate (SDS) as a surfactant to facilitate nanobubble generation. The size of the nanobubbles in this method depends on factors such as agitation speed, time, temperature of the solution, dissolved gas content, and water chemistry. Wu et al. report yielding bulk nanobubbles with a mean diameter of $500 \mathrm{~nm}$ and a lifetime of about 24 hours. ${ }^{118}$ One of BHIA cell's main advantages compared with other cavitation techniques is the ability to have real-time control of agitation speeds, which significantly affects the size of the produced nanobubbles. Wu et al. found that the increase in agitation speed with surfactant agents generates smaller bubbles with higher concentrations. ${ }^{118}$

4.1.3.2 Hydrodynamic cavitation by using centrifugal multiphase pump (CMP). To begin the nanobubble fabrication process using a CMP, atmospheric air is delivered into the suction part of a CMP that is pre-filled with $40 \mathrm{~L}$ of DI water. This creates a mixture of gas and water inside the container. Different gas flows are used to facilitate the dissolution of air in water. When the air is completely dissolved in water, the

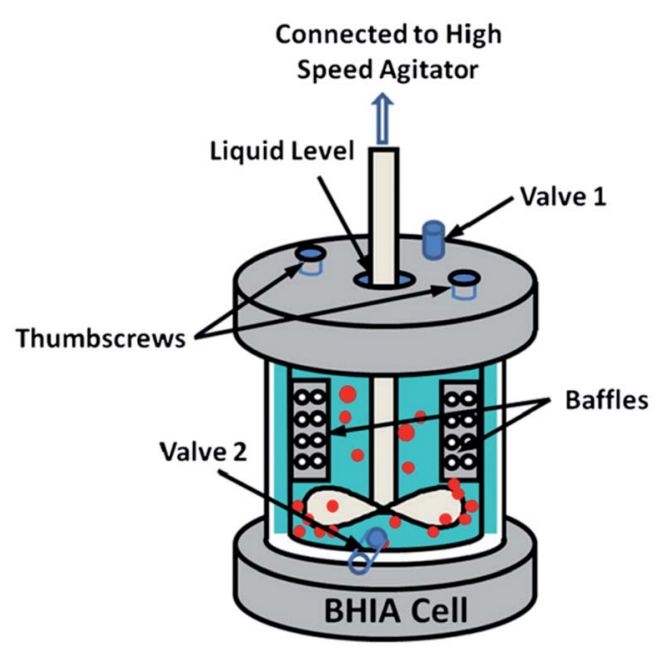

Fig. 9 Baffled high-intensity agitation (BHIA) cell used by Wu et al. to generate bulk nanobubbles. After introducing the solution into a baffled cell, the rotating impeller connected to a high-speed agitator creates hydrodynamic cavitation leading to the generation of bulk nanobubbles. Valves 1 and 2 are used to take samples for the characterization of the generated bulk nanobubbles. The thumbscrews are placed to tightly seal the cap cell to the cell body. ${ }^{118}$ 
mixture is pumped through a needle valve for nanobubble generation. ${ }^{\mathbf{1 2 0 - 1 2 2}}$ Fig. 10 illustrates the CMP setup used by Etchepare et $a l .{ }^{119}$ to generate nanobubbles within the range of 150-200 nm. The highest concentration they reach is $4 \times 10^{9}$ $\mathrm{mL}^{-1} .^{119}$ However, since they do not use any surfactants in the solution, the lifetime of the generated nanobubbles is short. Calgaroto et al. also report producing bulk nanobubbles with the smallest size of 150-180 nm. They utilize SDS and Flotigam EDA $3 \mathrm{~B}$ as ionic and cationic surfactants, respectively, to improve the lifetime of the nanobubbles. ${ }^{123}$

\subsubsection{Hydrodynamic cavitation on a microfluidic platform.} In this method, nanobubble formation results from the hydrodynamic cavitation that occurs within a microfluidic chamber. As water is passed through the device, nucleation of nanobubbles occurs due to the high shear stresses and sudden channel expansion at the junction of the device (Fig. 11). This phenomenon can be explained with Bernoulli's mechanical energy conservation principle, where the decrease in local pressure below the vapor pressure causes cavitation and the formation of tiny bubbles in the liquid. ${ }^{12}$ The setup includes an inlet reservoir of non-degassed pure water connected to the Y-type microfluidic chip where cavitation occurs. The resulting bubbles are then passed through a heat exchanger to decrease the temperature and finally pumped to the outlet for collection. The mean bubble diameter reported by Nirmalkar et al. remains constant at approximately $130 \mathrm{~nm}$, with a considerable population lasting up to 3 months. Their findings also reveal a positive correlation between bubble number density and the pump's operating pressure at the inlet. ${ }^{12}$

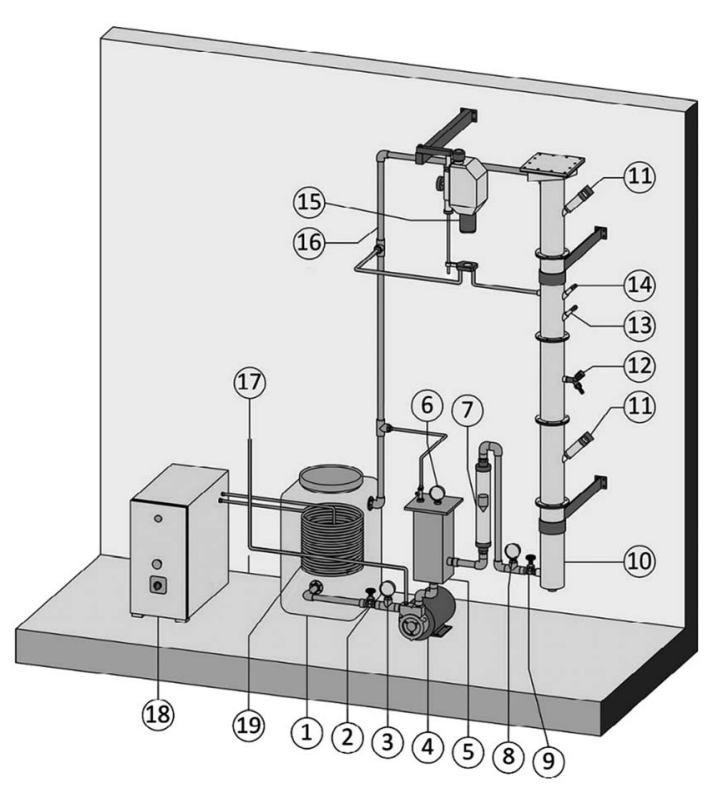

Fig. 10 The generation of bulk nanobubbles in a semi-continuous system using a centrifugal multiphase pump (CMP). Atmospheric air is injected into the CMP (item 4 in the figure) and passed through the pump impellers. The impeller's shear forces cause a multiphasic (air/ liquid) flow, which is then subjected to different operating pressures to saturate the air in the water. Then, the saturated water is forced through the needle valve (item 9) for bubble generation through hydrodynamic cavitation. ${ }^{119}$

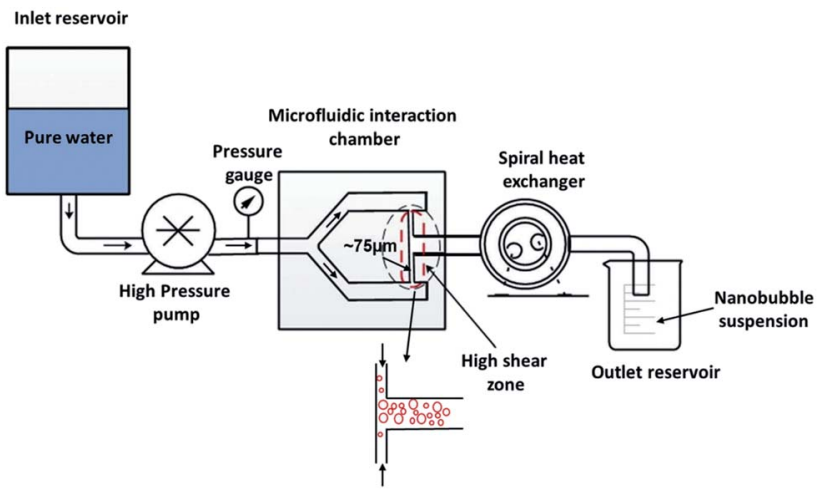

Fig. 11 The experimental setup with the integration of a $Y$-type microfluidic cell used by Nirmalkar et al. for the generation of bulk nanobubbles. ${ }^{12}$

4.1.3.4 Compression-decompression. In this technique, developed by several different researchers, a high pressure gas is injected into a specific volume of a liquid using a needle. ${ }^{\mathbf{1 2 4 - 1 2 7}}$ A plunger is employed to reach an optimal amount of mixing between the gas and the liquid. When the pressure of the mixture is reduced to atmospheric pressure, the gas inside the liquid starts to form bubbles. Repeating the compressiondecompression of the liquid-gas mixture leads to generation of bulk nanobubbles. Recently, Jin et al. utilize this method to produce bulk nanobubbles with a mean size of about $280 \mathrm{~nm}$ and size distribution of 100-800 nm. ${ }^{124}$

4.1.4 Surface electrostatic nanobubble formation. A novel method that is introduced by Ghaani et al. is using an electric field to generate nanobubbles. ${ }^{128}$ The experimental setup for this technique consists of a stainless-steel container that contains deionized water (Fig. 12). A connected tube to the container injects a specific gas (methane or oxygen) to the container until achieving a gas saturated water. A fixed electric field about $12 \mathrm{kV} \mathrm{m}^{-1}$ is applied to the water through the attached wires on the bottom of the container. The authors hypothesize that applying an electrostrictive force via an electric field induces regions of negative pressure in a dielectric liquid, leading to the formation of nanobubbles. The mean diameter of the nanobubbles increase from about $220 \mathrm{~nm}$ to $300 \mathrm{~nm}$ after four months. ${ }^{128}$

4.1.5 Using graphene oxide sheets. If a surface exists when two miscible solvents which have different gas solubility are mixed with each other, gas supersaturation nucleation happens on the surface. Jannesari et al. utilize this phenomenon to make bulk nanobubbles. ${ }^{129}$ As seen in Fig. 13, the two miscible solvents are warm water and $\mathrm{NaCl}$ solution, saturated with cold nitrogen. Graphene oxide (GO) sheets are used as the nucleation surfaces for bulk nanobubbles. GO sheets are synthesized by a technique known as Hummers' method. Using a micro vortex platform helped the nucleated nanobubbles on the surface of the GO sheets to detach and become bulk nanobubbles. The diameter of the bulk nanobubbles is between $320-920 \mathrm{~nm}$, with the mean diameter around $545 \mathrm{~nm}^{129}$

4.1.6 Shirasu-porous-glass (SPG) membranes. Kukizaki et al. introduce a setup, employing a Shirasu-porous-glass membrane, to generate bulk nanobubbles. ${ }^{130}$ The primary 
A

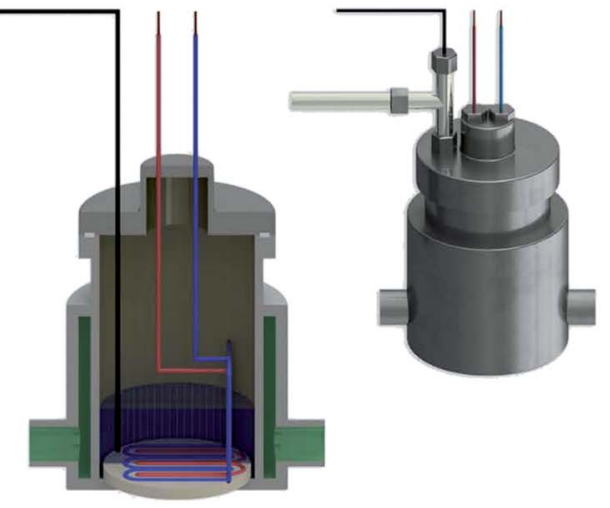

B

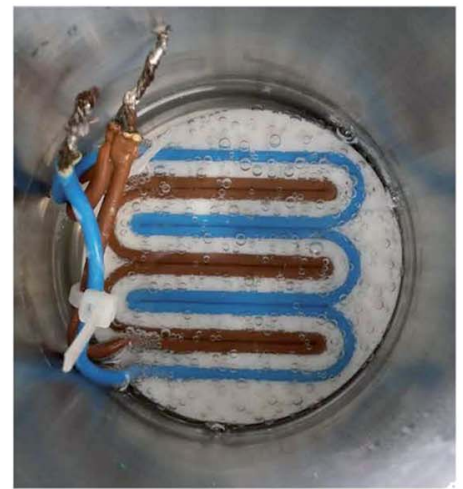

Fig. 12 Pressure vessel rig used for creation of surface electrostatics nanobubbles. (A) Cross section of the pressure vessel that include the gas supplier, distribution terminal the pressure cell and the temperature regulation jacket. (B) DC current supply set up via sheath covered wires in a 3 dimensional printed plastic. ${ }^{128}$

section of this setup is the membrane module, which houses a tubular glass membrane with submicron diameter pores through which water-SDS solution passes. Pressurized air is introduced into the module outside of the glass membrane and forced into the flowing solution by transmembrane pressure differential, resulting in nanobubble formation. Drag forces acting on the bubble detach it from the pore and introduce it into the bulk solution (Fig. 14). As a result, bulk nanobubbles form in the fluid. The mean diameter of the produced nanobubbles with this setup is $360-720 \mathrm{~nm}$. The porous glass membrane is made of a mixture of sodium carbonate, calcium carbonate, magnesium oxide, and boric acid. To fabricate the tubular glass, a molding process is utilized and hydrochloric acid solution is used to make micro-holes on the surface of the glass. The nanobubbles generated with this method have better size monodispersity compared to the other methods. ${ }^{130}$

4.1.7 Ultrasonic irradiation. When water is irradiated by ultrasound, fine bubbles are generated at nucleation sites, grow to about resonance size under acoustic pressure fluctuations, and collapse. The generation of bulk nanobubbles through ultrasonic irradiation reported by Yasuda et al. involves

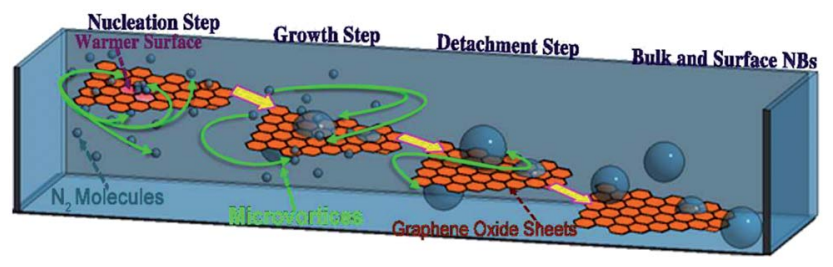

Fig. 13 The schematic illustration of four different steps for bulk nanobubbles creation through microvortices and graphene oxide sheets. In the first step (nucleation step), gas supersaturation nucleation caused by two miscible solvents with different gas solubility forms nanobubbles on a graphene sheet (growth step). Warm water and cold nitrogen saturated $\mathrm{NaCl}$ are the mentioned solvents. In the growth step, nanobubbles on the surface of the graphene sheet grow until reaching their critical size and detach from the surface (detachment step) and form bulk nanobubbles inside the solution (bulk and surface nanobubbles step). ${ }^{129}$ a Langevin transducer attached to stainless steel vibration plates at the bottom of a vessel, and a circulating water bath to maintain constant temperature in the sample. ${ }^{131}$ The transducer is controlled by a signal generator driven by a power amplifier to maintain a constant sinusoidal wave throughout the bubble generation process. During production, ultrapure water is sonicated by the transducer which caused creation of submicron bubbles through acoustic cavitation (Fig. 15). They test various ultrasound frequencies, from $22 \mathrm{kHz}$ to $1 \mathrm{MHz}$, and reveal a trend of increasing nanobubble concentration with decreasing ultrasound frequency, while the effect of frequency on the mode nanobubble diameter is insignificant. They also study the effect of ultrasonic power on nanobubble number concentration, and show that the concentration increases with ultrasonic power. They report that the mode diameter of the generated bulk nanobubbles is within $90-100 \mathrm{~nm}$ for all

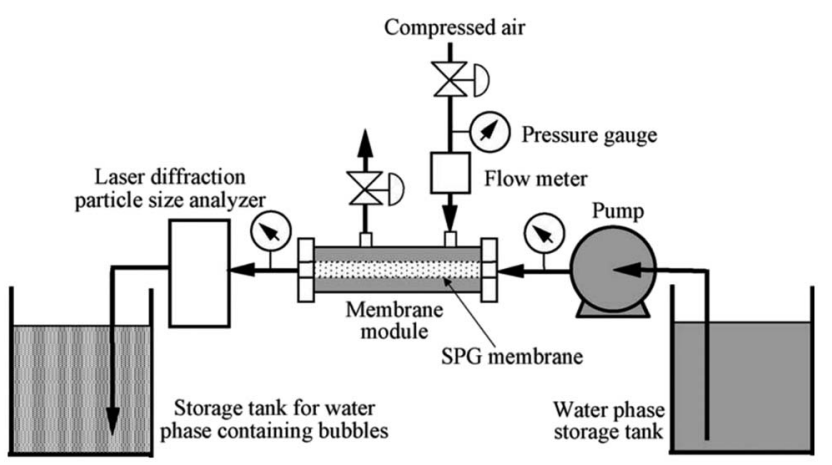

Fig. 14 Shirasu-porous-glass (SPG) membrane used to produce bulk nanobubbles. The process starts by pumping a solution consists of water and sodium dodecyl sulphate (SDS) from the water phase storage tank into the membrane module. Then, the compressed air is purged into the membrane through the membrane holes to form bulk nanobubbles inside the membrane. Then, the produced bulk nanobubbles are collected in the storage tank. A flowmeter, a pressure gauge, and a laser diffraction particle size analyzer are used to measure the flow of the water-SDS solution, air pressure, and size of the generated nanobubbles, respectively. ${ }^{130}$ 
a)

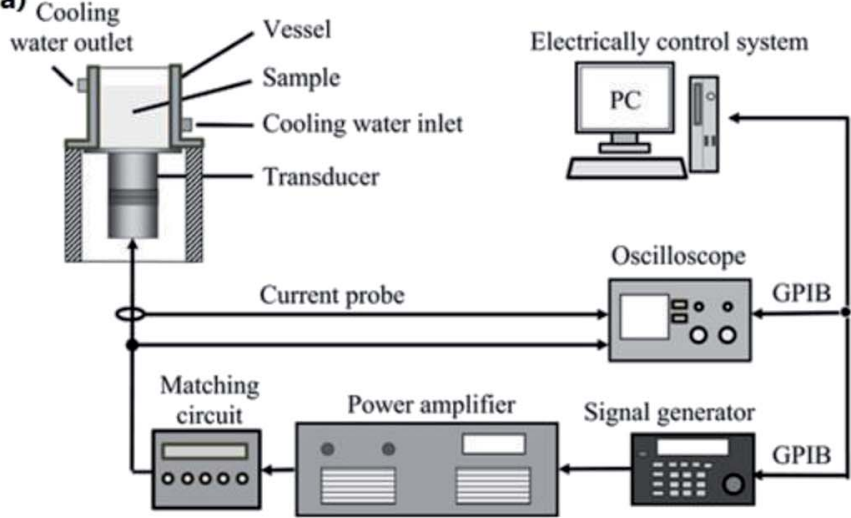

b)

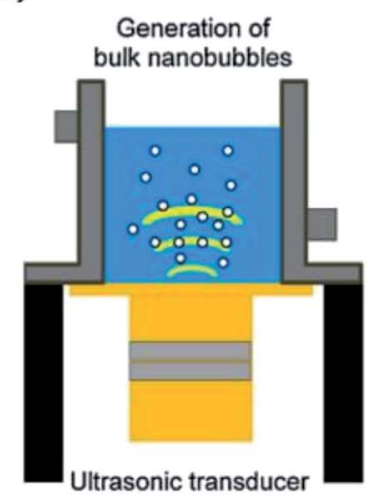

Fig. 15 Ultrasonic irradiation apparatus that are used for generation of bulk nanobubbles. (a) Langevin transducer controlled by a signal generator attached to stainless-steel vibration plates. (b) Illustration of the generation of bulk nanobubbles. ${ }^{131}$

conditions, and the concentration of the bubbles increases with increasing the irradiation time, asymptotically reaching a value of $1.5 \times 10^{9} \mathrm{~mL}^{-1} .131$

4.1.8 Microbubble fragmentation by ultrasound. Fragmentation of microbubbles into nanobubbles is another technique proposed by Huynh et al. ${ }^{132}$ They first generate microbubbles using an agitation method and then convert them to bulk nanobubbles by exposing them to low-frequency ( 1 $\mathrm{MHz}$ ), high duty-cycle (50\%) ultrasound (Fig. 16). The shell of the microbubbles is porphyrin-lipid, and the core gas is perfluorocarbon. They show that the lipid-shell nanobubbles have an excellent multimodal imaging capability, effectively producing ultrasound, photoacoustic, and fluorescence imaging enhancement. The lifetime of the generated nanobubbles is about 22 days, measured by NanoSight LM10. The nanobubbles produced with this method are polydisperse and have a size distribution of $5-500 \mathrm{~nm}^{.132}$

4.1.9 Generation via nanoporous membrane. Ma et al. use a nanoporous alumina membrane to generate bulk nanobubbles. ${ }^{133}$ The alumina membrane is composed of nanoholes with a mean diameter of about $100 \mathrm{~nm}$. The researchers employ the anodization technique as a simple, inexpensive, and efficient method to fabricate the membrane. ${ }^{133}$ To produce bulk nanobubbles, they introduce gas through the nanoporous membrane into a cell filled with DI water for 1 hour at a gas pressure of 1.5 atm. ${ }^{133}$ The gas passes through the nanopores, and after pinching off from the nanopores in DI water, bulk nanobubbles form. This approach produces $\mathrm{CO}_{2}$ bulk nanobubbles within the diameter range of 50-200 $\mathrm{nm}$. Moreover, to investigate the stability of the bulk nanobubbles, the authors use other gases, including nitrogen, oxygen, helium, and argon. ${ }^{133}$ They find that under the same conditions, $\mathrm{CO}_{2}$ nanobubbles are smaller than the other gases due to their higher solubility in water. The produced bulk nanobubbles have a concentration of approximately $6 \times 10^{7}$ $\mathrm{mL}^{-1}$, which is acceptable for several in vivo and in vitro biomedical applications. However, the technique they introduce, like other methods mentioned earlier, cannot precisely control nanobubble diameter. Moreover, the lifetime and polydispersity index of the generated bulk nanobubbles have not been investigated. ${ }^{\mathbf{1 3 3}}$

\subsection{Chemical methods}

4.2.1 Electrolysis. The generation of nanobubbles via an electrochemical cell is another method that has been used over the past few years. As shown in Fig. 17, the setup is composed of two electrodes that are fixed in a cell and connected to an external power supply. By applying a voltage to the electrodes, the electrical current from anode to cathode electrolyzes the water in the cell. The result of the electrolysis reaction is the formation of oxygen nanobubbles in the anode side of the cell. ${ }^{135,136}$ Kikuchi et al. report the production of bulk oxygen nanobubbles with an initial size of

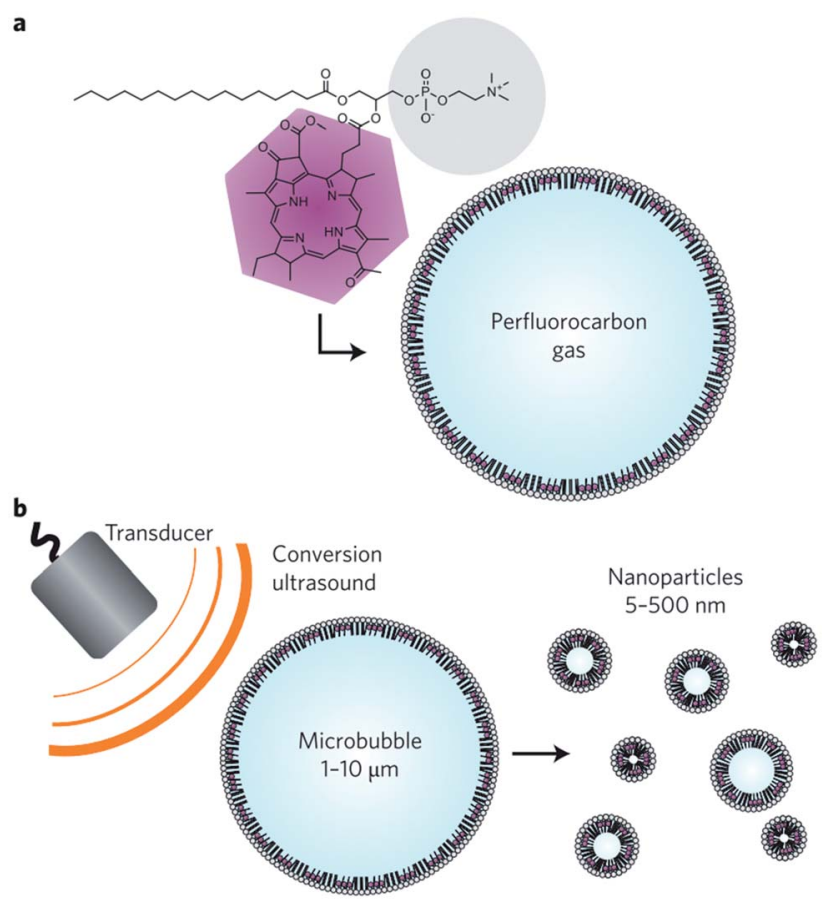

Fig. 16 Bulk nanobubbles generation via fragmentation of microbubbles. (a) A microbubble consists of bacteriochlorophyll-lipid and perfluorocarbon gas. (b) Production of bulk nanobubbles through applying low-frequency ultrasound to the bacteriochlorophyll-lipid shell microbubbles. ${ }^{132}$ 


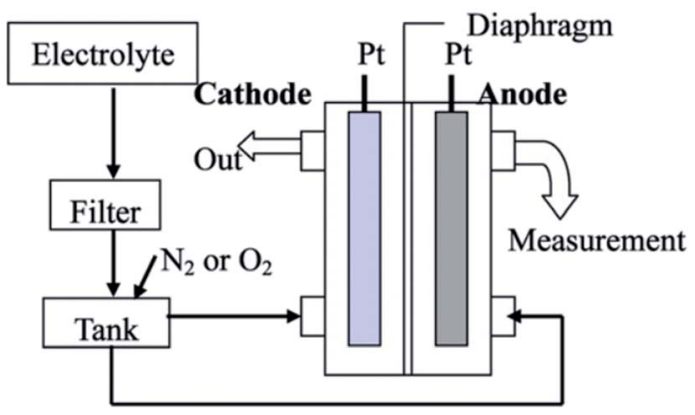

Fig. 17 Production of bulk nanobubbles through the electrolysis technique. An electrolyte solution passes from a filter with $100 \mathrm{~nm}$ holes before being merged with gas $\left(\mathrm{O}_{2}\right.$ or $\left.\mathrm{N}_{2}\right)$ in the tank. Then, the mixed solution with gas in the tank transports to the chamber where the electrodes are placed. The electric current passing from the electrode anode to the cathode electrode electrolyzes the water inside the chamber. ${ }^{134}$

$30 \mathrm{~nm}$. Three days after the generation of the bulk nanobubbles, the final size of the nanobubbles increases to $250 \mathrm{~nm}$, and the nanobubbles gradually disappear as a result of dissolution of oxygen from the bubbles. ${ }^{\mathbf{1 3 4}}$

4.2.2 Plasma. Sato et al. use plasma to generate nanobubbles. ${ }^{138}$ In their experimental setup, one side of a platinum wire electrode is placed inside a water container, and the other side is connected to a power supply. ${ }^{137}$ A platinum ring electrode connected to the power supply, and a ground electrode is placed under the bottom of the water vessel. A voltage within the range of -3.5 to $-5.5 \mathrm{kV}$ with a frequency of $10 \mathrm{kHz}$ is applied to the electrode that generate plasma emission at the tip of the wire electrode. They explain that underwater plasma emission creates gas channels along "streamer discharges", which collapse to form bulk nanobubbles with the mean diameter of $120 \mathrm{~nm}$ and the size distribution of 50-400 nm (Fig. 18). ${ }^{137}$

\subsection{Discussion}

Altogether, principal characteristics, including concentration, polydispersity index, real-time control on bubble size, and the lifetime of the generated bulk nanobubbles distinguish the

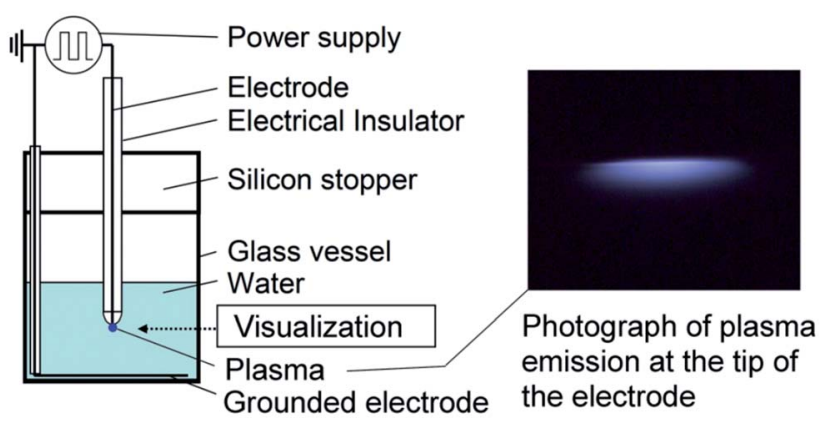

Fig. 18 Schematic of the experimental setup to form bulk nanobubbles via plasma generation in a specific volume of water. The plasma forms between the electrode tip placed in the water and the grounded electrode after turning on the high-voltage power supply. ${ }^{137}$ production methods from each other. In this section, we delve into the advantages and disadvantages of the existing bulk nanobubble manufacturing techniques and examine their biomedical applications.

In general, agitation-based techniques produce bulk nanobubbles with high concentrations, which can then be diluted to lower concentrations for specific applications. Namely, de Leon et al. are able to reach a concentration of up to $10^{11} \mathrm{~mL}^{-1} \cdot{ }^{113}$ The gas used in these methods are typically perfluorocarbons, which have very low solubility in a aqueous phase. ${ }^{139}$ As a result, the lifetime of bulk nanobubbles produced via agitation is prolonged. However, high polydispersity index and lack of real-time control on the size of the nanobubbles are the foremost drawbacks of these agitation-based methods.

Bulk nanobubbles generated via agitation methods have been employed in molecular imaging as ultrasound and photoacoustic contrast agents. ${ }^{2,39,42,47}$ Biomedical applications whose results do not depend on a low nanobubble polydispersity index can benefit from the generation methods based on agitation.

Another conventional method to manufacture bulk nanobubbles is using the hydrodynamic cavitation technique. The main advantage of this method is its ability to produce moderately highly concentration bulk nanobubbles $\left(10^{8}\right.$ to $10^{9}$ $\left.\mathrm{mL}^{-1}\right) .{ }^{120,121}$ A downside of employing the hydrodynamic method is the large footprint of the generation setups required for this technique. So far, nanobubbles produced through this technique have only been employed in non-medical applications, including mineral and food processing. Nevertheless, the ability of this method to fabricate concentrated bulk nanobubbles may assist in biomedical applications that demand highly-concentrated nanobubbles in large quantities.

One of the practices to avoid Ostwald ripening, which decreases the lifetime and causes an undesirable high polydispersity index, is coating a nanobubble surface with a stabilizing shell. Functionalizing a nanobubble surface with a lipid layer could improve the nanobubble stability and monodispersity by reducing the surface tension, resulting a decrease in the Laplace pressure according to the Young-Laplace equation. ${ }^{140}$ In diagnostic and therapeutic applications such as in vivo ultrasound imaging, ultrasound-mediated gene delivery, and tumour ablation, coating the nanobubbles in a stabilizing shell prolongs the circulation time, which in turn extends the time window for contrast-enhanced ultrasound imaging. ${ }^{\mathbf{1 4 1 , 1 4 2}}$ Consequently, the manufacturing techniques that produce solely non-lipid shell nanobubbles might be less reliable for biomedical applications that require stable monodisperse bulk nanobubbles for optimal efficacy. These methods include graphene oxide sheets, ultrasonic irradiation, and plasma.

Among the nanobubble generation techniques described in this review, electrolysis produces the most concentrated bulk nanobubbles $\left(10^{18}\right.$ to $\left.10^{21} \mathrm{~mL}^{-1}\right) \cdot{ }^{134,135}$ This advantage is important in dentistry since enhancing nanobubble concentration induces a significant boost in stem cell proliferation, leading to efficiency improvement. However, in this method, it is not technically possible to precisely control the size of the nanobubbles. Moreover, the produced nanobubbles are not 
stable; Kikuchi et al. report a significant change in the size of the produced nanobubbles via electrolysis from $30 \mathrm{~nm}$ to $250 \mathrm{~nm}$ within three days. ${ }^{134}$

On the contrary, the surface electrostatic method may generate reasonably stable nanobubbles. Ghaani et al. use this technique to form bulk nanobubbles with a mean diameter of around $220 \mathrm{~nm}$, increasing to $300 \mathrm{~nm}$ after four months. ${ }^{\mathbf{1 2 8}}$ However, since the authors have not reported nanobubble concentration and polydispersity index, it is unknown whether such nanobubbles can be used in medical applications. One factor that can make a difference in whether bulk nanobubbles can be utilized in applications is the type of gas encapsulated inside the nanobubbles. In tumour hypoxia treatment and wound healing, oxygen bulk nanobubbles are required to secure a satisfactory level of oxygen in the damaged hypoxic tissues. ${ }^{62,69}$ To this end, generation via nanoporous membrane might be an ideal approach to fill the nucleus of nanobubbles with oxygen. ${ }^{133}$ However, in this method, the size of the nanobubbles is dictated by the diameter of the membrane nanopores. As such, this method is not appropriate for applications that need real-time control over the bubble size.

Lastly, microbubble fragmentation can be employed as a simple and straightforward technique to manufacture lipidshell bulk nanobubbles. ${ }^{\mathbf{1 3 2}}$ However, since the ultrasonic radiation intensity is not uniform over the sample containing the microbubbles, a homogeneous size distribution cannot be obtained, deteriorating the polydispersity index. Huynh et al. use the nanobubbles produced using this technique as contrast agents in photoacoustic imaging. ${ }^{132}$ To summarize, based on the requirements of the applications of nanobubbles, an ideal nanobubble production method would have a high throughput, and make monodisperse and high-concentration nanobubbles that are stable and can be functionalized. Though all the techniques we have reviewed fulfils one or more of these criteria, not a single method realizes the entire criteria set.

Microfluidics has the potential to be utilized as a transformative technique for nanobubble generation. This is because the requirements of size control, high concentration, and monodispersity have been addressed by microfluidics researchers in making bulk microdroplets and microbubbles. ${ }^{87,143-146}$ The major challenge for microfluidics would be to further scale down the bubble size, so that nanoscale bubbles can be generated. In Section 5, we discuss how microfluidics can contribute to producing monodisperse bulk nanobubbles. Table 2 summarizes the specifications of the available production techniques.

\section{Nanobubble generation opportunities for microfluidics}

Bulk nanobubbles with the characteristics of controllable size, monodispersity, and high concentration, further the development of several important biomedical applications. In this section, we first outline the existing techniques use microfluidics to produce bulk nanobubbles. Then, we propose two additional ideas to engineer microfluidic devices that generate bulk nanobubbles with the characteristics that are highly desirable in many biomedical applications.

\subsection{Existing technique 1: microfluidic atomization}

In the atomization method described by Peyman et al. ${ }^{\mathbf{1 4 8}}$ a microfluidic device consisting of a central gas inlet channel and two opposing liquid inlet channels form a flow focusing geometry, allowing for atomization-like flow focusing production of both micro- and nanobubbles. In the experiment illustrated in Fig. 19, a perfluorocarbon gas is injected in the gas inlet and a lipid solution is injected into the liquid inlet channels, to create a flow focusing production stream. Shear stresses and pressure changes at the channel expansion create an atomization-like phenomenon, producing microbubbles and a fine spray of particles that are nanometers in size. The particles consist of a mixed population of nanobubbles and perfluorocarbon particles.

Subsequently, a passive separation method is used to separate the nanoparticles and microbubbles. The separation method relies on the intrinsic buoyancy of the bubbles. Nanoparticle tracking analysis is used to characterize the nanobubbles and the authors find a high number concentration of particles produced, around $10^{10} \mathrm{~mL}^{-1}$, for particles within the range of 100-300 $\mathrm{nm}$. The authors do not report the polydispersity index of the produced nanobubbles, and the ability of this technique to control the size of the nanobubbles is not investigated.

Some of the main advantages of using this microchipmicrospray approach include its ease of operation, high reproducibility, and low manufacturing costs. Experiments for targeted drug delivery demonstrate this technique as a rapid, single-step, nanobubble functionalization method. ${ }^{148}$

\subsection{Existing technique 2: microfluidic shrinkage}

Our group has recently reported a method for generating monodisperse lipid-shell nanobubbles using a microfluidic device. ${ }^{\mathbf{1 4 9}}$ In this method, monodisperse microbubbles are generated in a microfluidic flow-focusing platform. Shrinkage enables the transformation of microbubbles into nanobubbles. To achieve this, the gas core consists of a mixture of two gases, one of which is highly soluble in the liquid phase, and another gas that is weakly soluble. After the generation of microbubbles, the highly soluble gas component dissolves in the liquid phase, leaving the weakly soluble component as the gas core of a much smaller bubble (Fig. 20). ${ }^{\mathbf{1 4 9}}$

The final diameter of the shrunk bubbles can be adjusted by modifying design parameters, including core gas composition and the width of the flow focusing junction. This method offers real-time control over the monodispersity, size, and production rate of the resulting nanobubbles, simply by adjusting the flow conditions, such as gas pressure and liquid flow rate, within the device. It is also the first known method for creating monodisperse nanobubbles, with a favourable polydispersity index of 0.11 , at a sufficient throughput for biomedical applications. ${ }^{\mathbf{1 4 9}}$

A drawback of this method is the existence of an upper limit of the bubble concentration, due to the nature of the shrinkage process. To achieve maximum concentration, spherical bubbles must assume a "close-packed" arrangement. The number of bubbles per unit volume, pre-shrinkage, determines the 
Table 2 Summary of each nanobubble generation method

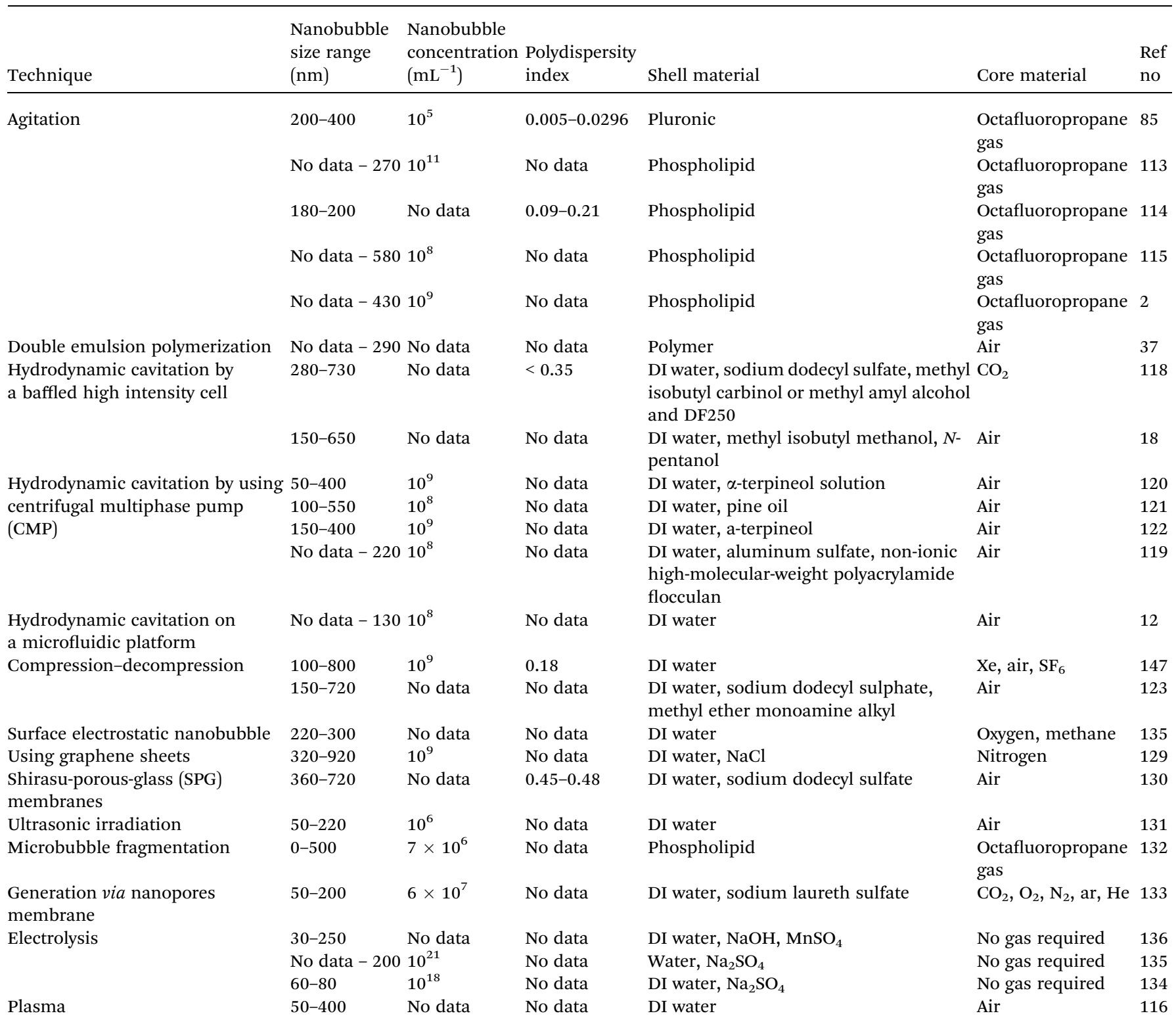

maximum possible concentration, as shrunk bubbles cannot possibly assume the same arrangement. Increasing the concentration limit would require reducing the initial microbubble diameter to accommodate more bubbles per unit volume. This would necessitate more complex device fabrication protocols as initial microbubble diameter is dependent on the width of the flow-focusing orifice. We report concentrations up to $10^{8} \mathrm{~mL}^{-1}$ and show that this is acceptable for ultrasound contrast enhancement, but this does not currently allow for the flexibility provided by, for example, the agitation method, which can yield concentrations up to $10^{11} \mathrm{~mL}^{-1} \cdot{ }^{149}$

\subsection{Nanobubble generation possibilities with microfluidics}

The two aforementioned studies on utilizing microfluidics to produce bulk nanobubbles demonstrate that, despite some possible drawbacks, microfluidics may be able to address the limitations of conventional production methods. For instance, the design recently published by our group provides the functionality of real-time nanobubble size control, with a low polydispersity index, which addresses a major challenge for almost all bulk nanobubble manufacturing methods. ${ }^{149}$ Furthermore, using a microfluidic flow-focusing design facilitates the possibility of forming nanobubbles with combinations of various gases, by simply supplying different gases, and their mixtures, as the dispersed phase. This feature of microfluidics provides flexibility for many nanobubble-based biomedical applications.

In the sections below, we present two additional potential microfluidics research projects that may contribute to improving the size range of and/or throughput of microfluidic nanobubble generation.

5.3.1 Reducing microfluidic flow focusing junction width using DRIE. A ubiquitous microfluidic geometry utilized to 
a)

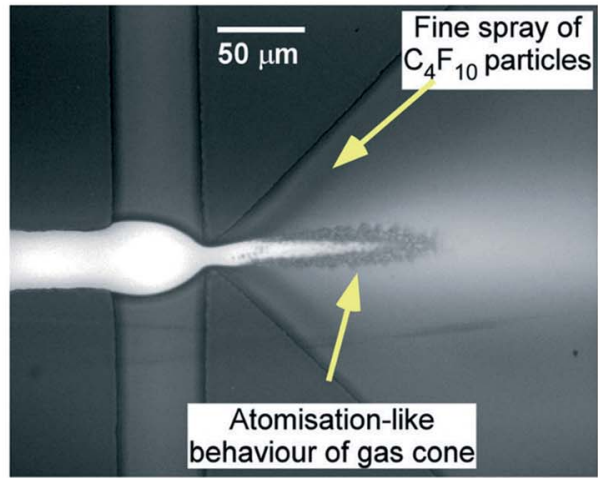

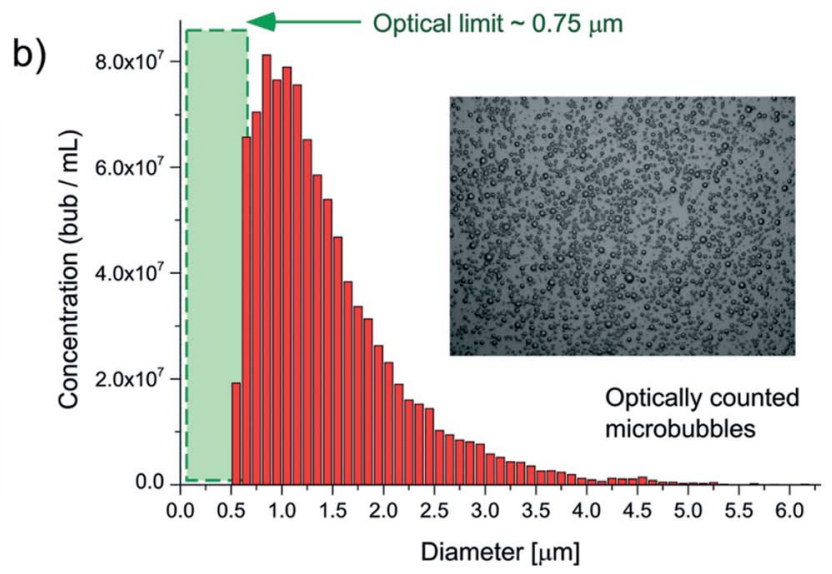

Fig. 19 (a) Microscopic image of a microfluidic flow focusing geometry with a 3D expansion, illustrating the atomization-like production of microbubbles, and fine-spray production of nanoparticles. (b) Histogram depicting an optically counted concentration distribution of particles, reported by Peyman et al. The optical limit of $0.75 \mu \mathrm{m}$ is reached within the green shaded region. ${ }^{148}$

generate bulk microbubbles is the flow focusing design. This design is typically built using the typical method of patterning PDMS, and then bonding the PDMS to a glass slide. ${ }^{150-161}$ As shown in Fig. 21, in the flow focusing technique, two liquid sheath flows from the side channels cut off the gas flow from the central channel at the orifice location. As a result, the gas phase breaks up into microbubbles surrounded by the liquid continuous phase. With this geometry, the diameter of microbubbles depends on the liquid flow rate, gas flow rate, and orifice width. Eqn (3) shows the relationship between the diameter of a microbubble and the mentioned parameters, ${ }^{162}$

$$
\frac{d_{\mathrm{b}}}{D} \propto\left(\frac{Q_{\mathrm{g}}}{Q_{\mathrm{l}}}\right)^{0.4}
$$

where $d_{\mathrm{b}}$ is the diameter of a bubble, $D$ is the width of the orifice and $Q_{\mathrm{g}}$ and $Q_{\mathrm{l}}$ are the gas and liquid flow rates, respectively. As stated by eqn (3), decreasing the width of the orifice causes a reduction in the microbubble diameter. However, due to the limitations of the soft lithography process, which is a common method to fabricate microfluidic devices, achieving orifices with widths less than $5 \mu \mathrm{m}$ is an arduous and demanding task in the fabrication of PDMS-based microstructures. Generating nanobubbles by decreasing the orifice width to less than $5 \mu \mathrm{m}$ has

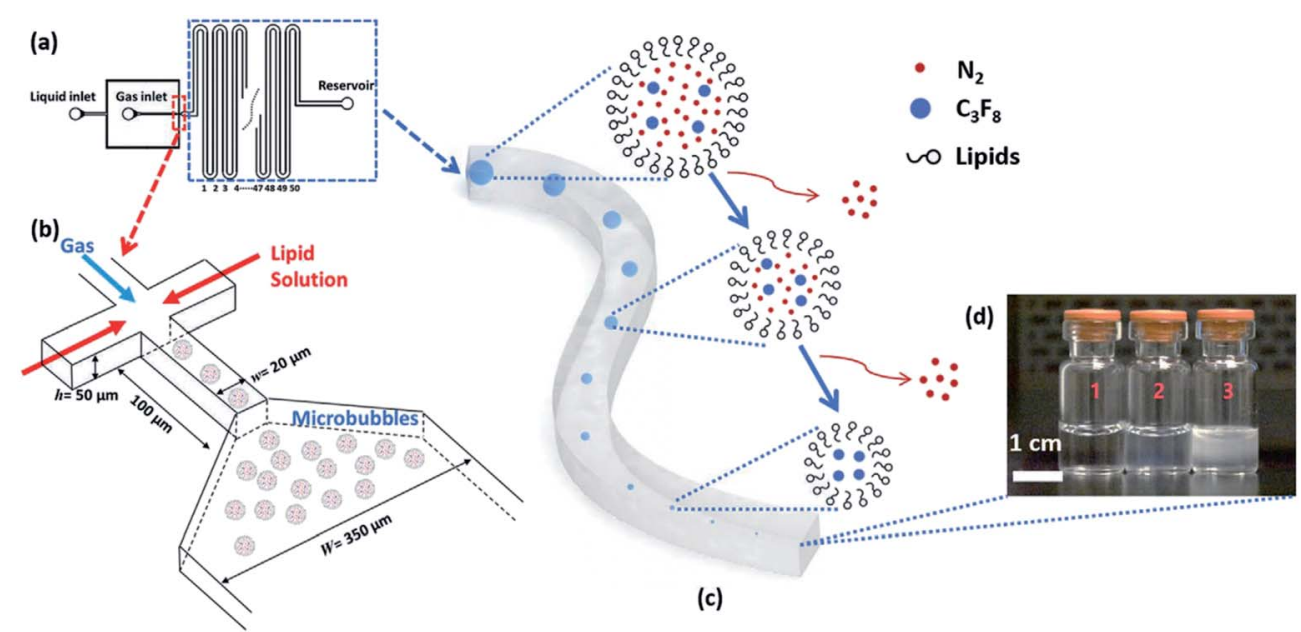

Fig. 20 A microfluidic nanobubble generator that is recently reported by our group. (a) Top-view schematic diagram of the microfluidic chip, showing liquid (lipid solution), gas and reservoir inlets. The serpentine structure of the design facilitates the tracking of microbubble shrinkage into nanobubbles by a microscope. (b) The flow-focusing section of the microfluidic chip to generate monodisperse microbubbles. The width and length of the orifice are $20 \mu \mathrm{m}$ and $100 \mu \mathrm{m}$, respectively. Following the generation of the microbubbles, the shrinkage process begins with the entry of the microbubbles into the serpentine microchannel. The width of this microchannel is $350 \mu \mathrm{m}$, and the height of all channels in the device is $50 \mu \mathrm{m}$. (c) The gradual outflow of nitrogen from the cores of microbubbles and dissolution into the aqueous phase leads to microbubble shrinkage. In contrast, $\mathrm{C}_{3} \mathrm{~F}_{8}$ remains inside the bubble's core due to its low solubility. (d) Three different samples are taken from the reservoir inlet. Sample 1 is the control group consisting of lipid solution, and samples 2 and 3 contain bulk nanobubbles with mean diameters of 100 nm and $200 \mathrm{~nm}$, respectively. ${ }^{149}$ 


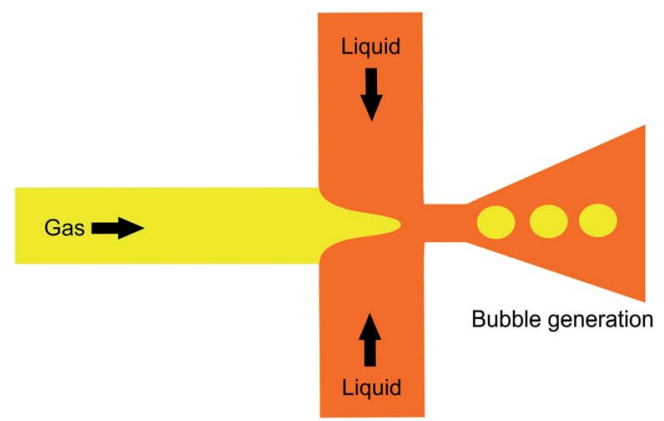

Fig. 21 Top-view schematic diagram of a planar flow focusing design to produce bubbles.

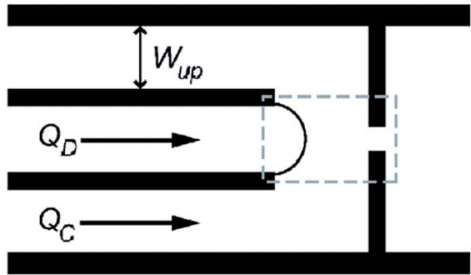

(a)

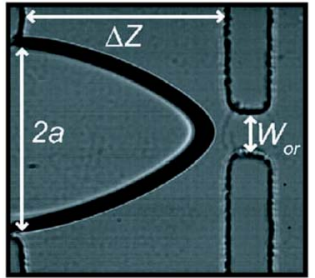

(b)
Fig. 22 Microbubble production by flow focusing. (a) A schematic drawing, and (b) microscopic image of flow focusing geometries. In (a), $Q_{D}, Q_{C}$, and $W_{\text {up }}$ represent the gas flow rate, liquid flow rate, and distance between the device wall and the gas channel, respectively. In (b), the orifice width, the gas flow channel width, and the junction-toorifice distance is shown by $W_{O R}, 2 a$, and $\Delta Z$, respectively. ${ }^{154}$

not been discussed in other papers so far. We believe one of the reliable solutions to this matter is replacing the PDMS substrate that comprises microchannels with silicon and utilizing standard micro/nano machining processes in the Micro Electro Mechanical Systems (MEMS) field to fabricate orifices with ultra-high aspect ratios. Deep Reactive Ion Etching (DRIE) is one of the conventional MEMS procedures to fabricate fine micro/nano structures such as micro/nanochannels. For example, Parasuraman et al. fabricate silicone trenches with
$99.49 \mu \mathrm{m}$ height and $800 \mathrm{~nm}$ width, which shows the capability of the DRIE technique to fabricate high aspect ratio micro/nano structures. ${ }^{163}$

Alternatively, a reduction in bubble diameter can also be achieved by increasing the liquid flow rate to transition from the geometry-controlled regime, in which bubbles have a size almost equal to the orifice width, to the dripping regime. This regime can be achieved by changing the capillary number, which is defined as

$$
\mathrm{Ca}=\frac{\mu_{0} G a}{\gamma_{\mathrm{EQ}}}=\frac{\mu_{0} a \Delta V}{\gamma_{\mathrm{EQ}} \Delta Z}=\frac{\mu_{0} a Q_{\mathrm{C}}}{\gamma_{\mathrm{EQ}} h \Delta Z}\left[\frac{1}{W_{\mathrm{OR}}}-\frac{1}{2 W_{\mathrm{UP}}}\right],
$$

where $\mu_{0}$ is the dynamic viscosity of the liquid, $G$ is the elongation rate, $a$ is the half-width of the channel that carries the gas, and $y_{\mathrm{EQ}}$ is the equilibrium surface tension. The elongation rate can be defined as $G=\left(V_{\mathrm{c}}-V_{\mathrm{u}}\right) / \Delta Z$, where $V_{\mathrm{c}}$ and $V_{\mathrm{u}}$ are the largest and the smallest fluid velocity at the orifice, respectively, and $\Delta Z$ is the liquid channel width. In eqn (4), $\Delta V=V_{\mathrm{c}}-V_{\mathrm{u}}$ can be written as $Q_{\mathrm{C}} / h \times\left(1 / W_{\mathrm{OR}}-1 / 2 W_{\mathrm{UP}}\right)$, where $Q_{\mathrm{C}}$ is the liquid flow rate, $h$ is the height of the channels, and $W_{\mathrm{OR}}$ and $W_{\mathrm{UP}}$ are the width of the orifice and the channels that carry the liquid, respectively (Fig. 22). ${ }^{154}$

It is worth mentioning that in miniaturizing the orifice, some critical issues should be considered. During operation, blockage of the orifice is one of the problems that may arise due to reducing the orifice width.

To prevent the probable clogging, the solution (continuous phase) should be filtered through syringe filters with pores smaller than the orifice width. Moreover, in designing the flowfocusing microfluidic device, filtering micro channels should be considered to prevent orifice clogging. Fig. 23 briefly illustrates the proposed idea of producing nanobubbles via reducing the orifice width in a bulk microbubble generator microfluidic device with a flow-focusing design.

5.3.2 Parallelized nanobubble production by embedding a nanoporous membrane. Another approach to produce bulk monodisperse nanobubbles, with increased throughput, is to embed a silicone nanoporous membrane with uniformly sized a)

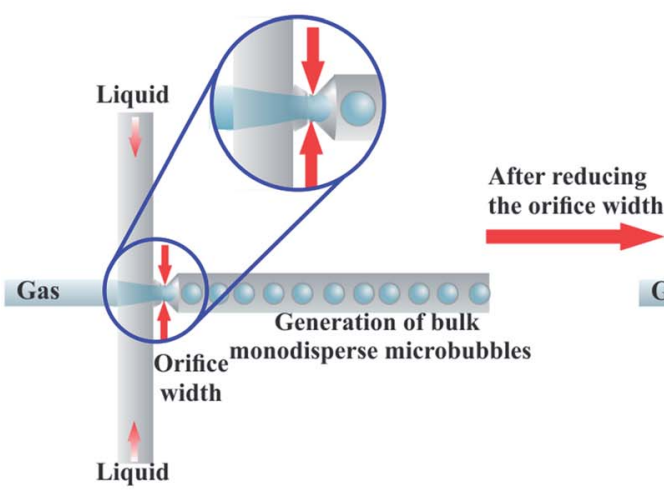

b)

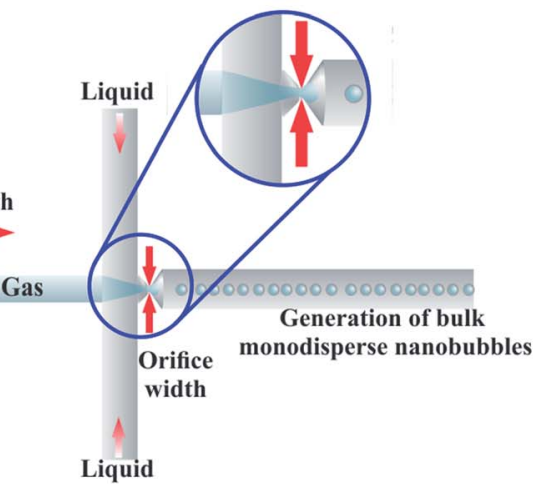

Fig. 23 Generation of bulk microbubbles through (a) flow-focusing technique in a microfluidic device and (b) replacing the PDMS substrate with silicone and reducing the orifice width to achieve bulk nanobubbles. 


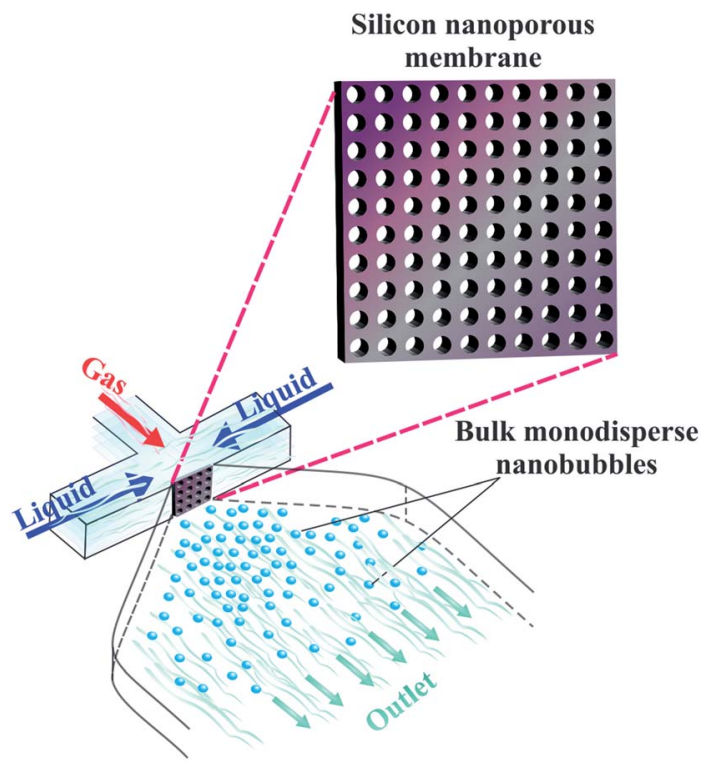

Fig. 24 Production of bulk monodisperse nanobubbles via an embedded silicone nanoporous membrane in a PDMS microfluidic chip.

pores, into a microfluidic planar flow focused design. Fig. 24 shows a schematic diagram of the proposed idea.

In this design, the continuous liquid phase and the dispersed gas phase are forced toward the nanopores by creating a pressure gradient. The liquid flow from the lateral microchannels squeezes the gas through the nanoporous membrane location, passing the nanoholes. As a result, nanobubbles should form and pinch off at each membrane hole, into the outlet channel. Since the nanopores are uniformly sized, the generated bulk nanobubbles should have a low polydispersity index.

This design should facilitate higher nanobubble generation throughput. Since bubble shrinkage is not needed in this design, the concentration limit in our recent work could be drastically improved. ${ }^{149}$ In addition to reducing the polydispersity index, it may be possible to control the size of the nanobubbles here by simply changing the gas and liquid pressure. This approach should also be manufacturable using conventional soft lithography processes. $^{\mathbf{1 6 4 , 1 6 5}}$ To embed the nanoporous silicon membrane inside the chip, we propose the standard oxygen plasma process. Moreover, ready-made nanoporous membranes can be utilized instead of fabricating a nanoporous membrane via anodization techniques, to minimize manufacturing complexity.

\section{Conclusion}

Nanobubbles can be used in many applications, from industrial to medical. Indeed, bulk nanobubbles are already utilized in some therapeutic and diagnostic applications because of their unique acoustic and transport properties. Consequently, various methods have been introduced to generate bulk nanobubbles. However, despite the progress made in the field of nanobubble generation, there are still some important drawbacks that limit nanobubble widespread utilization. In many of the biomedical applications of bulk nanobubbles, such as imaging, better nanobubble monodispersity would potentially significantly improve outcomes. However, the currently utilized bulk nanobubble generation methods generally make polydisperse nanobubbles, and lack realtime size control capabilities.

Microfluidics has emerged as a tool that has mass production capability, and precise fluid flow controllability. With additional innovations, microfluidics may be developed to overcome existing nanobubble production challenges, to make better nanobubbles for biomedical applications. Evidence of this claim can be seen in the number of published papers in recent years in the field of nanodroplet and bulk microbubble production. We hope this review demonstrates that nanobubble generation is a highly impactful area of research, and that microfluidics researchers have an important role to play in developing the next generation of highly tunable, monodisperse, and high concentration nanobubbles. In biomedical applications, that could improve diagnostic and therapeutic modalities, and ultimately, patient outcomes. We also propose two microfluidic methods to address the drawbacks of the current bulk nanobubble generation techniques.

\section{Conflicts of interest}

The authors declare no conflict of interest.

\section{Acknowledgements}

SSHT is thankful for research funding from the Canada Foundation for Innovation (CFI 36687 and 36442), and the Natural Sciences and Engineering Research Council (NSERC) of Canada Discovery grants program (RGPIN-2019-04618). MCK is thankful for research funding support from the Canada Foundation for Innovation (CFI 30994), NSERC Discovery Grant (RGPIN2017-06496), and the NSERC/CIHR Collaborative Health Research Projects (NSERC/CIHR - CPG 146484). The authors would like to thank Katherine Chan from Ryerson University for editing early drafts of the manuscript, and Amir Mohammad Seif from Tehran University for assistance in making the graphical contents of Section 5 .

\section{References}

1 B. D. Johnson and R. C. Cooke, Generation of stabilized microbubbles in seawater, Science, 1981, 213(4504), 209-211.

2 T. Yin, P. Wang, R. Zheng, B. Zheng, D. Cheng, X. Zhang, et al., Nanobubbles for enhanced ultrasound imaging of tumors, Int. J. Nanomed., 2012, 7, 895-904.

3 P. Attard, M. P. Moody and J. W. G. Tyrrell, Nanobubbles: The big picture, Phys. Stat. Mech. Appl., 2002, 314(1-4), 696-705.

4 J. Zhu, H. An, M. Alheshibri, L. Liu, P. M. J. Terpstra, G. Liu, et al., Cleaning with bulk nanobubbles, Langmuir, 2016, 32(43), 11203-11211.

5 J. N. Meegoda, S. Aluthgun Hewage and J. H. Batagoda, Stability of nanobubbles, Environ. Eng. Sci., 2018, 35(11), 1216-1227. 
6 J. L. Parker, P. M. Claesson and P. Attard, Bubbles, cavities, and the long-ranged attraction between hydrophobic surfaces, J. Phys. Chem., 1994, 98(34), 8468-8480.

7 G. S. Manning, On the thermodynamic stability of bubbles, immiscible droplets, and cavities, Phys. Chem. Chem. Phys., 2020, 22(31), 17523-17531.

8 P. S. Epstein and M. S. Plesset, On the stability of gas bubbles in liquid-gas solutions, J. Chem. Phys., 1951, 19(2), 256.

9 M. Alheshibri, J. Qian, M. Jehannin and V. S. J. Craig, A History of nanobubbles, Langmuir, 2016, 32(43), 1108611100.

$10 \mathrm{~S}$. H. Oh and J. M. Kim, Generation and stability of bulk nanobubbles, Langmuir, 2017, 33(15), 3818-3823.

11 N. Nirmalkar, A. W. Pacek and M. Barigou, On the existence and stability of bulk nanobubbles, Langmuir, 2018, 34(37), 10964-10973.

12 N. Nirmalkar, A. W. Pacek and M. Barigou, Interpreting the interfacial and colloidal stability of bulk nanobubbles, Soft Matter, 2018, 14(47), 9643-9656.

13 N. Nirmalkar, A. W. Pacek and M. Barigou, Bulk Nanobubbles from Acoustically Cavitated Aqueous Organic Solvent Mixtures, Langmuir, 2019, 35, 2188-2195.

14 A. J. Jadhav and M. Barigou, Bulk nanobubbles or not nanobubbles: that is the question, Langmuir, 2020, 36(7), 1699-1708.

15 G. Ferraro, A. J. Jadhav and M. Barigou, A Henry's law method for generating bulk nanobubbles, Nanoscale, 2020, 12(29), 15869-15879.

16 D. Rak, M. Ovadová and M. Sedlák, (Non)existence of bulk nanobubbles: the role of ultrasonic cavitation and organic solutes in water, J. Phys. Chem. Lett., 2019, 10(15), 42154221.

17 V. Leroy and T. Norisuye, Investigating the existence of bulk nanobubbles with ultrasound, ChemPhysChem, 2016, 27872790.

18 M. H. Alheshibri, PhD thesis, Australian National University, 2019.

19 M. Alheshibri and V. S. J. Craig, Armoured nanobubbles; ultrasound contrast agents under pressure, J. Colloid Interface Sci., 2019, 537, 123-131.

20 C. Chen, J. Li and X. Zhang, The existence and stability of bulk nanobubbles: a long-standing dispute on the experimentally observed mesoscopic inhomogeneities in aqueous solutions, Commun. Theor. Phys., 2020, 72(3), 037601.

21 T. Temesgen, T. T. Bui, M. Han, T. i. Kim and H. Park, Micro and nanobubble technologies as a new horizon for watertreatment techniques: a review, Adv. Colloid Interface Sci., 2017, 246, 40-51.

22 K. K. Thi Phan, T. Truong, Y. Wang and B. Bhandari, Nanobubbles: fundamental characteristics and applications in food processing, Trends Food Sci. Technol., 2020, 95, 118-130.

23 K. K. T. Phan, T. Truong, Y. Wang and B. Bhandari, Formation and stability of carbon dioxide nanobubbles for potential applications in food processing, Food Eng. Rev., 2020, 15, 1-2.

24 B. W. Davis, J. T. Diep and S. Jose, US Pat., US 9642591 B2, 2017.

25 A. d. Leon, R. Perera, P. Nittayacharn, M. Cooley, O. Jung and A. A. Exner, Ultrasound contrast agents and delivery systems in cancer detection and therapy, Adv. Canc. Res., 2018, 139, 57-84.

26 A. A. Exner and M. C. Kolios, Bursting microbubbles: how nanobubble contrast agents can enable the future of medical ultrasound molecular imaging and image-guided therapy, Curr. Opin. Colloid Interface Sci., 2021, 54, 101463.

27 D. V. B. Batchelor, F. J. Armistead, N. Ingram, S. A. Peyman, J. R. Mclaughlan, P. L. Coletta, et al., Nanobubbles for therapeutic delivery: production, stability and current prospects, Curr. Opin. Colloid Interface Sci., 2021, 54, 101456.

28 E. P. Favvas, G. Z. Kyzas, E. K. Efthimiadou and A. C. Mitropoulos, Bulk nanobubbles, generation methods and potential applications, Curr. Opin. Colloid Interface Sci., 2021, 54, 101455.

29 W. Cui, S. Tavri, M. J. Benchimol, M. Itani, E. S. Olson, H. Zhang, et al., Biomaterials neural progenitor cells labeling with microbubble contrast agent for ultrasound imaging in vivo, Biomaterials, 2013, 34(21), 4926-4935.

30 C. R. Anderson, X. Hu, H. Zhang and J. Tlaxca, Ultrasound molecular imaging of tumor angiogenesis with an integrin targeted microbubble contrast agent, Invest. Radiol., 2011, 46(4), 215-224.

31 B. Jin, M. Lin, M. You, Y. Zong, M. Wan and F. Xu, Microbubble embedded with upconversion nanoparticles as a bimodal contrast agent for fluorescence and ultrasound imaging, Nanotechnology, 2015, 26(34), 345601.

32 T. M. Krupka, L. Solorio, R. E. Wilson, H. Wu, N. Azar and A. A. Exner, Formulation and characterization of echogenic lipid-pluronic nanobubbles, Mol. Pharm., 2010, 7(1), 49-59.

33 F. Conversano, R. Franchini, A. Lay-ekuakille and S. Casciaro, In vitro evaluation and theoretical modeling of the dissolution behavior of a microbubble contrast agent for ultrasound imaging, IEEE Sens. J., 2012, 12(3), 496-503.

34 V. Sboros, C. Moran, S. Pye and W. McDicken, An in vitro study of a microbubble contrast agent using a clinical ultrasound imaging system, Phys. Med. Biol., 2003, 49(1), 159.

35 A. K. Iyer, G. Khaled, J. Fang and H. Maeda, Exploiting the enhanced permeability and retention effect for tumor targeting, Drug Discov. Today, 2006, 11(17-18), 812-818.

36 H. Yang, W. Cai, L. Xu, X. Lv, Y. Qiao, P. Li, et al., Nanobubble-Affibody: novel ultrasound contrast agents for targeted molecular ultrasound imaging of tumor, Biomaterials, 2015, 37, 279-288.

37 M. R. Böhmer, C. H. T. Chlon, B. I. Raju, C. T. Chin, T. Shevchenko and A. L. Klibanov, Focused ultrasound and microbubbles for enhanced extravasation, J. Control. Release, 2010, 148(1), 18-24. 
38 J. N. Marsh, K. C. Partlow, D. R. Abendschein, M. J. Scott, M. Gregory and S. A. W. Lanza, Molecular imaging with targeted perfluorocarbon nanoparticles: quantification of the concentration dependence of contrast enhancement for binding to sparse cellular epitopes, Ultrasound Med. Biol., 2007, 33(6), 950-958.

39 C. Kim, R. Qin, J. S. Xu, L. V. Wang and R. Xu, Multifunctional microbubbles and nanobubbles for photoacoustic and ultrasound imaging, Biomed. Opt., 2015, 15, 13-15.

40 R. H. Perera, H. Wu, P. Peiris, C. H. Bs, A. B. Bs, H. Z. Bs, et al., Improving performance of nanoscale ultrasound contrast agents using N,N-diethylacrylamide stabilization, Nanomed. Nanotechnol. Biol. Med., 2016, 1-9.

$41 \mathrm{M}$. Kuriakose and M. A. Borden, Microbubbles and Nanodrops for photoacoustic tomography, Colloid Interface Sci., 2021, 101464.

42 R. X. Xu, Multifunctional microbubbles and nanobubbles for photoacoustic imaging, Contrast Media Mol. Imaging, 2011, 6(5), 401-411.

43 F. J. Bodera, M. J. Moore, Y. Wang, A. C. D. Leon, E. Abenojar, A. A. Exner, et al., Nanobubble facilitated optoporation and photoacoustic imaging of BT-474 breast cancer cells, IEEE Int. Ultrason. Symp., 2018, 1-4.

44 M. Xu, L. V. Wang, M. Xu and L. V. Wang, Photoacoustic imaging in biomedicine, Rev. Sci. Instrum., 2006, 77(4), 041101.

45 X. Yang, E. W. Stein, S. Ashkenazi and L. V. Wang, Nanoparticles for photoacoustic imaging, Wiley Interdiscip. Rev. Nanomed. Nanobiotechnol., 2009, 1(4), 360-368.

46 P. Beard, Biomedical photoacoustic imaging, Interface Focus, 2011, 1(4), 602-631.

47 W. Wu, Z. Zhang, L. Zhuo, L. Zhou, P. Liu, Y. He, et al., Ultrasound molecular imaging of acute cellular cardiac allograft rejection in rat with T-cell-specific nanobubbles, Transplantation, 2013, 96(6), 543-549.

48 A. S. Thakor and S. S. Gambhir, Nanooncology: the future of cancer diagnosis and therapy, Ca-Cancer J. Clin., 2013, 63(6), 395-418.

49 J. Liu, Y. Chen, G. Wang, Q. Lv, Y. Yang, J. Wang, et al., Biomaterials ultrasound molecular imaging of acute cardiac transplantation rejection using nanobubbles targeted to T lymphocytes, Biomaterials, 2018, 162, 200-207.

50 Z. Yu, M. Hu, Z. Li, D. Xu and L. Zhu, Anti-G250 nanobodyfunctionalized nanobubbles targeting renal cell carcinoma cells for ultrasound molecular imaging, Nanotechnology, 2020, 31(20), 205101.

51 R. H. Perera, A. D. Leon, X. Wang, Y. Wang, P. Peiris, E. Abenojar, et al., Real time ultrasound molecular imaging of prostate cancer with PSMA-targeted nanobubbles, Nanomed. Nanotechnol. Biol. Med., 2020, 28, 102213.

52 A. Oishi, H. Shimokawa, E. Sakaniwa, M. Takahashi, M. Miyashin and S. Arakawa, Oxygen and air nanobubbles in water inhibit proliferation of dental follicle stem cells in vitro, J. Dent. Health Oral Disord. Ther., 2018, 9(6), 460462.

53 S. Arakawa, M. Sugisawa and A. Leewananthawet, Application of Ozone Nanobubble Water (ONBW) to PeriImplantitis Treatment, Dentistry, 2017, 7(12), 1-6.

54 S. Hayakumo, S. Arakawa, Y. Mano and Y. Izumi, Clinical and microbiological effects of ozone nano-bubble water irrigation as an adjunct to mechanical subgingival debridement in periodontitis patients in a randomized controlled trial, Clin. Oral Invest., 2013, 17(2), 379-388.

55 S. Keely, L. E. Glover, C. F. Macmanus, E. L. Campbell, M. M. Scully, G. T. Furuta, et al., Selective induction of integrin $\beta \mathrm{i}$ by hypoxia-inducible factor: implications for wound healing, Faseb. J., 2009, 23(5), 1338-1346.

56 R. Kumari, L. B. Willing, J. K. Krady, S. J. Vannucci and I. A. Simpson, Impaired wound healing after cerebral hypoxia - ischemia in the diabetic mouse, J. Cereb. Blood Flow Metab., 2007, 24(7), 710-718.

57 W. X. Hong, M. S. Hu, M. Esquivel, G. Y. Liang, R. C. Rennert, A. Mcardle, et al., The role of hypoxiainducible factor in wound healing, Adv. Wound Care, 2014, 3(5), 390-399.

58 J. H. A. Niinikoski, Clinical hyperbaric oxygen therapy, wound perfusion, and transcutaneous, World J. Surg., 2004, 28(3), 307-311.

59 E. Uhl, A. Sirsjö, T. Haapaniemi and G. N. G. Nilsson, Hyperbaric oxygen improves wound healing in normal and ischemic skin tissue, Plast. Reconstr. Surg., 1994, 93(4), 835-841.

60 S. Bhutani and G. Vishwanath, Hyperbaric oxygen and wound healing, Indian J. Plast. Surg., 2019, 45(2), 316.

61 S. Juma and C. G. Brito, Transobturator tape (TOT): two years follow-Up, Neurourol. Urodyn., 2007, 41, 37-41.

62 L. R. Sayadi, D. A. Banyard, M. E. Ziegler, Z. Obagi, J. Prussak, M. J. Klopfer, et al., Topical oxygen therapy \& micro/nanobubbles: a new modality for tissue oxygen delivery, Int. Wound J., 2018, 15(3), 363-374.

63 J. G. Rajendran and K. A. Krohn, PET imaging for tumor hypoxia: characterizing the tumor and guiding treatment, Positron Emission Tomography, 2006, 359-374.

64 A. K. Wong, V. J. Reid, F. Gewalli and B. J. Mehrara, The effect of hyperbaric oxygen treatment on squamous cell cancer growth and tumor hypoxia, Ann. Plast. Surg., 2008, 60(1), 81-88.

65 W. Huang, M. Shen, H. Chen, S. Lin, W. Chiang, P. Wu, et al., Monocytic delivery of therapeutic oxygen bubbles for dual-modality treatment of tumor hypoxia Wen-Chia, J. Control. Release, 2015, 220, 738-750.

$66 \mathrm{~K}$. Graham and E. Unger, Overcoming tumor hypoxia as a barrier to radiotherapy, chemotherapy and immunotherapy in cancer treatment, Int. J. Nanomed., 2018, 6049.

67 A. Schroeder, J. Kost and Y. Barenholz, Ultrasound, liposomes, and drug delivery: principles for using ultrasound to control the release of drugs from liposomes, Chem. Phys. Lipids, 2009, 162(1-2), 1-6. 
68 Y. Cao, Y. Chen, T. Yu, Y. Guo, F. Liu, Y. Yao, et al., Drug release from phase-changeable nanodroplets triggered by Low-intensity focused ultrasound, Theranostics, 2018, 8(5), 1327.

69 R. Song, S. Peng, Q. Lin, M. Luo, H. Y. Chung, Y. Zhang, et al., pH-responsive oxygen nanobubbles for spontaneous oxygen delivery in hypoxic tumors, Langmuir, 2019, 35(31), 10166-10172.

70 R. Suzuki, Y. Oda, N. Utoguchi and K. Maruyama, Progress in the development of ultrasound-mediated gene delivery systems utilizing nano- and microbubbles, J. Control. Release, 2011, 149(1), 36-41.

71 P. D. Robbins and S. C. Ghivizzani, Viral vectors for gene therapy, Pharmacol. Ther., 1998, 80(1), 35-47.

72 C. Pellow, J. Tan, E. Chérin, C. E. M. Demore, G. Zheng and D. E. Goertz, High frequency ultrasound nonlinear scattering from porphyrin nanobubbles, Ultrasonics, 2021, 110, 106245.

73 H. Kida, K. Nishimura, K. Ogawa, A. Watanabe and L. B. Feril, Nanobubble mediated gene delivery in conjunction with a hand-held ultrasound scanner, Front. Pharmacol., 2020, 11, 1-10.

74 A. Aoi, Y. Watanabe, S. Mori, M. Takahashi, G. Vassaux and T. Kodama, Herpes simplex virus thymidine kinasemediated suicide gene therapy using nano/microbubbles and ultrasound, Ultrasound Med. Biol., 2008, 34(3), 425-434.

75 Y. Negishi, Y. Endo, T. Fukuyama, R. Suzuki, T. Takizawa, D. Omata, et al., Delivery of siRNA into the cytoplasm by liposomal bubbles and ultrasound, J. Control. Release, 2008, 132(2), 124-130.

76 Z. Liu, J. Zhang, Y. Tian, L. Zhang, X. Han, Q. Wang, et al., Targeted delivery of reduced graphene oxide nanosheets using multifunctional ultrasound nanobubbles for visualization and enhanced photothermal therapy, Int. J. Nanomed., 2018, 13, 7859.

77 E. M. Knavel and C. L. Brace, Tumor ablation: common modalities and general practices, Tech. Vasc. Interv. Radiol., 2013, 16(4), 192-200.

78 H. Y. Lee, H. Rhim, M. W. Lee, Y. Kim, D. Choi, M. J. Park, et al., Early diffuse recurrence of hepatocellular carcinoma after percutaneous radiofrequency ablation: analysis of risk factors, Eur. Radiol., 2013, 23(1), 190-197.

79 A. Zerbini, M. Pilli, D. Laccabue, G. Pelosi, A. Molinari, E. Negri, et al., Radiofrequency thermal ablation for hepatocellular carcinoma stimulates autologous NK-cell response, Gastroenterology, 2010, 138(5), 1931-1942.

80 N. N. Zhang, W. Lu, X. J. Cheng, J. Y. Liu, Y. H. Zhou and F. Li, High-powered microwave ablation of larger hepatocellular carcinoma: evaluation of recurrence rate and factors related to recurrence, Clin. Radiol., 2015, 70(11), 1237-1243.

81 R. H. Perera, L. Solorio, H. Wu, M. Gangolli, E. Silverman, C. Hernandez, et al., Nanobubble ultrasound contrast agents for enhanced delivery of thermal sensitizer to tumors undergoing radiofrequency ablation, Pharm. Res., 2013, 31(6), 1407-1417.
82 R. Suzuki, Y. Oda, D. Omata, N. Nishiie, R. Koshima, Y. Shiono, et al., Tumor growth suppression by the combination of nanobubbles and ultrasound, Cancer Sci., 2016, 107(3), 217-223.

83 X. Zhang, Y. Zheng, Z. Wang, S. Huang, Y. Chen, W. Jiang, et al., Methotrexate-loaded PLGA nanobubbles for ultrasound imaging and synergistic targeted therapy of residual tumor during HIFU ablation, Biomaterials, 2014, 35(19), 5148-5161.

84 Y. Yao, K. Yang, Y. Cao, X. Zhou, J. Xu and J. Liu, Comparison of the synergistic effect of lipid nanobubbles and SonoVue microbubbles for high intensity focused ultrasound thermal ablation of tumors, PeerJ, 2016, 4, e1716.

85 N. J. McDannold, I. Natalia and K. H. Vykhodtseva, Microbubble contrast agent with focused ultrasound to create brain lesions at low histologic study in rabbits, Radiology, 2006, 241(1), 95-106.

86 F. Calliada, R. Campani, O. Bottinelli and M. G. S. Anna Bozzini, Ultrasound contrast agents basic principles, Eur. J. Radiol., 1998, 27, S157-S160.

87 H. Wu, E. C. Abenojar, R. Perera and A. C. De Leon, Tianzhi an AAE. Time-intensity-curve analysis and tumor extravasation of nanobubble ultrasound contrast agents, Ultrasound Med. Biol., 2019, 45(9), 2502-2514.

88 L. R. Sayadi, D. A. Banyard, M. E. Ziegler, Z. Obagi, J. Prussak, M. J. Klopfer, et al., Topical oxygen therapy \& micro/nanobubbles: a new modality for tissue oxygen delivery, Int. Wound J., 2018, 15(3), 363-374.

89 I. Trapani, Adeno-associated viral vectors as a tool for large gene delivery to the retina, Genes, 2019, 10(4), 287.

90 J. Nathaniel, Gene delivery to human limbal stem cells using viral vectors, Hum. Gene Ther., 2019, 30(11), 13361348.

91 A. G. Therapy, A. A. Deviatkin, Y. A. Vakulenko, L. V. Akhmadishina and V. V. Tarasov, Emerging concepts and challenges in rheumatoid, Biomedicines, 2020, 8(1), 9.

92 W. I. Goldburg, Dynamic light scattering, Am. J. Phys., 1999, 67(12), 1152-1160.

93 R. Pecora, Dynamic light scattering measurement of nanometer particles in liquids, Nanoparticle Res., 2000, 2(2), 123-131.

94 J. Stetefeld, S. A. McKenna and T. R. Patel, Dynamic light scattering: a practical guide and applications in biomedical sciences, Biophys. Rev., 2016, 8(4), 409-427.

95 F. Eklund, M. Alheshibri and J. Swenson, ScienceDirect Differentiating bulk nanobubbles from nanodroplets and nanoparticles, Curr. Opin. Colloid Interface Sci., 2021, 53, 101427.

96 C. A. Sennoga, J. S. M. Yeh, J. Alter, E. Stride, P. Nihoyannopoulos, J. M. Seddon, et al., Evaluation of Methods for Sizing and Counting of Ultrasound Contrast Agents, Ultrasound Med. Biol., 2012, 38(5), 834-845.

97 C. Hernandez, E. C. Abenojar, J. Hadley, A. C. De Leon, R. Coyne, R. Perera, et al., Sink or float? Characterization of shell-stabilized bulk nanobubbles using a resonant 
mass measurement technique, Nanoscale, 2019, 11(3), 851855.

98 T. P. Burg, M. Godin, S. M. Knudsen, W. Shen, G. Carlson, J. S. Foster, et al., Weighing of biomolecules, single cells and single nanoparticles in fluid, Nature, 2007, 446(7139), 1066-1069.

99 J. Gross, S. Sayle, A. R. Karow, U. Bakowsky and P. Garidel, Nanoparticle tracking analysis of particle size and concentration detection in suspensions of polymer and protein samples: influence of experimental and data evaluation parameters, Eur. J. Pharm. Biopharm., 2016, 104, 30-41.

100 M. Hamed Alheshibri, Nanobubbles in Bulk, 2019.

101 V. Filipe, A. Hawe and W. Jiskoot, Critical evaluation of nanoparticle tracking analysis (NTA) by NanoSight for the measurement of nanoparticles and protein aggregates, Pharm. Res., 2010, 27(5), 796-810.

102 A. Malloy and B. Carr, Nanoparticle tracking analysis - The halo ${ }^{\mathrm{TM}}$ system, Part. Part. Syst. Char., 2006, 23(2), 197-204.

103 R. A. Dragovic, C. Gardiner, A. S. Brooks, D. S. Tannetta, D. J. P. Ferguson, P. Hole, et al., Sizing and phenotyping of cellular vesicles using Nanoparticle Tracking Analysis, Nanomed. Nanotechnol. Biol. Med., 2011, 7(6), 780-788.

104 X. Zhang, Q. Wang, Z. Wu and D. Tao, An experimental study on size distribution and zeta potential of bulk cavitation nanobubbles, Int. J. Miner., 2020, 27(2), 12-15.

$105 \mathrm{~J}$. C. Bravman and R. Sinclair, The preparation of cross-section specimens for transmission electron microscopy, J. Electron. Microsc. Tech., 1984, 1(1), 53-61.

106 N. Dellby, L. Krivaneka, D. Nellist, E. Batson and R. Lupini, Progress in aberration-corrected scanning transmission electron microscopy, J. Electron. Microsc., 2001, 50(3), 177-185.

107 A. A. Jr, F. D. Nobre and F. A. Costa, The spin-1 Ising spin glass: a renormalization-group approach The spin-1 Ising spin glass: a renormalization-group approach, J. Phys., 2000, 12(26), 5713.

108 G. D. Danilatos, Foundations of environmental scanning electron microscopy, Adv. Electron. Electron. Phys., 1988, 71, 109-250.

109 M. Li, L. Tonggu, X. Zhan, T. L. Mega and L. Wang, Cryo-EM visualization of nanobubbles in aqueous solutions, Langmuir, 2016, 32(43), 11111-11115.

110 C. Hernandez, S. Gulati, G. Fioravanti, P. L. Stewart and A. A. Exner, Cryo-EM visualization of lipid and polymerstabilized perfluorocarbon gas nanobubbles - a step towards nanobubble mediated drug delivery, Sci. Rep., 2017, 7(1), 1-8.

111 F. Eklund, M. Alheshibri and J. Swenson, ScienceDirect differentiating bulk nanobubbles from nanodroplets and nanoparticles, Curr. Opin. Colloid Interface Sci., 2021, 53, 101427.

112 H. Jing, W. Cheng, S. Li, B. Wu, X. Leng, S. Xu, et al., Novel cell-penetrating peptide-loaded nanobubbles synergized with ultrasound irradiation enhance EGFR siRNA delivery for triple negative Breast cancer therapy, Colloids Surf. B Biointerfaces, 2016, 146, 387-395.
113 A. De Leon, R. Perera, C. Hernandez, M. Cooley, O. Jung, S. Jeganathan, et al., Contrast enhanced ultrasound imaging by nature-inspired ultrastable echogenic nanobubbles, Nanoscale, 2019, 11(33), 15647-15658.

114 W. Lin, X. Xie, J. Deng, H. Liu, Y. Chen, X. Fu, et al., Cellpenetrating peptide-doxorubicin conjugate loaded NGRmodified nanobubbles for ultrasound triggered drug delivery, J. Drug Target., 2015, 24(2), 134-146.

115 J. Tian, F. Yang, H. Cui, Y. Zhou, X. Ruan and N. Gu, A Novel Approach to Making the Gas-Filled Liposome Real: Based on the Interaction of Lipid with Free Nanobubble within the Solution, ACS Appl. Mater. Interfaces, 2015, 7(48), 26579-26584.

116 M. A. Wheatley, F. Forsberg, N. Dube, M. Patel and B. E. Oeffinger, Surfactant-stabilized contrast agent on the nanoscale for diagnostic ultrasound imaging, Ultrasound Med. Biol., 2006, 32(1), 83-93.

117 B. Luo, H. Liang, S. Zhang, X. Qin, X. Liu, W. Liu, et al., Novel lactoferrin-conjugated amphiphilic poly(aminoethyl ethylene phosphate)/poly(L-lactide) copolymer nanobubbles for tumor-targeting ultrasonic imaging, Int. J. Nanomed., 2015, 10, 5805.

118 C. Wu, K. Nesset, J. Masliyah and Z. Xu, Generation and characterization of submicron size bubbles, Adv. Colloid Interface Sci., 2012, 179, 123-132.

119 R. Etchepare, H. Oliveira, M. Nicknig, A. Azevedo and J. Rubio, Nanobubbles: Generation using a multiphase pump, properties and features in flotation, Miner. Eng., 2017, 112, 19-26.

$120 \mathrm{H}$. Oliveira, A. Azevedo and J. Rubio, Nanobubbles generation in a high-rate hydrodynamic cavitation tube, Miner. Eng., 2018, 116, 32-34.

121 A. Azevedo, R. Etchepare, S. Calgaroto and J. Rubio, Aqueous dispersions of nanobubbles: generation, properties and features, Miner. Eng., 2016, 94, 29-37.

122 A. Azevedo, R. Etchepare and J. Rubio, Raw water clarification by flotation with microbubbles and nanobubbles generated with a multiphase pump, Water Sci. Technol., 2017, 75(10), 2342-2349.

123 S. Calgaroto, K. Q. Wilberg and J. Rubio, On the nanobubbles interfacial properties and future applications in flotation, Miner. Eng., 2014, 60, 33-40.

124 J. Jin, R. Wang, J. Tang, L. Yang, Z. Feng, C. Xu, et al., Dynamic tracking of bulk nanobubbles from microbubbles shrinkage to collapse, Colloid. Surface. Physicochem. Eng. Aspect., 2020, 589, 124430.

125 J. Jin, Z. Feng, F. Yang and N. Gu, Bulk nanobubbles fabricated by repeated compression of microbubbles, Langmuir, 2019, 35(12), 4238-4245.

126 S. Ke, W. Xiao, N. Quan, Y. Dong, L. Zhang and J. Hu, Formation and Stability of Bulk Nanobubbles in Different Solutions, Langmuir, 2019, 35(15), 5250-5256.

127 Q. Wang, H. Zhao, N. Qi, Y. Qin, X. Zhang and Y. Li, Generation and stability of size-adjustable bulk nanobubbles based on periodic pressure change, Sci. Rep., 2019, 9(1), 1-9. 
128 M. R. Ghaani, P. G. Kusalik and N. J. English, Massive generation of metastable bulk nanobubbles in water by external electric fields, $S c i$. Adv., 2020, 6(14), 1-7.

129 M. Jannesari, O. Akhavan and H. R. Madaah Hosseini, Graphene oxide in generation of nanobubbles using controllable microvortices of jet flows, Carbon, 2018, 138, 8-17.

130 M. Kukizaki and M. Goto, Size control of nanobubbles generated from Shirasu-porous-glass (SPG) membranes, $J$. Membr. Sci., 2006, 281(1-2), 386-396.

131 K. Yasuda, H. Matsushima and Y. Asakura, Generation and reduction of bulk nanobubbles by ultrasonic irradiation, Chem. Eng. Sci., 2019, 195, 455-461.

132 E. Huynh, B. Y. C. Leung, B. L. Helfield, M. Shakiba, J. A. Gandier, C. S. Jin, et al., In situ conversion of porphyrin microbubbles to nanoparticles for multimodality imaging, Nat. Nanotechnol., 2015, 10(4), 325-332.

133 T. Ma, Y. Kimura, H. Yamamoto, X. Feng, A. Hirano-Iwata and M. Niwano, Characterization of bulk nanobubbles formed by using a porous alumina film with ordered nanopores, J. Phys. Chem. B, 2020, 124(24), 5067-5072.

134 K. Kikuchi, A. Ioka, T. Oku, Y. Tanaka, Y. Saihara and Z. Ogumi, Concentration determination of oxygen nanobubbles in electrolyzed water, J. Colloid Interface Sci., 2009, 329(2), 306-309.

135 A. V. Postnikov, I. V. Uvarov, N. V. Penkov and V. B. Svetovoy, Collective behavior of bulk nanobubbles produced by alternating polarity electrolysis, J. Mater. Chem. C, 2015, 3, 10715-10722.

136 A. V. Postnikov, I. V. Uvarov, M. V. Lokhanin and V. B. Svetovoy, High concentration of $\mathrm{H}_{2}$ and $\mathrm{O}_{2}$ nanobubbles in water electrolytes and their collective optical effect, AIP Conf. Proc., 2017, 1884.

137 T. Sato, T. Nakatani, T. Miyahara, S. Ochiai, M. Oizumi, H. Fujita, et al., Study on formation of plasma nanobubbles in water, J. Phys. Conf., 2015, 656(1), 012036.

138 T. Sato, T. Nakatani, T. Miyahara, S. Ochiai, M. Oizumi, H. Fujita, et al., Study on Formation of Plasma Nanobubbles in Water, J. Phys. Conf., 2015, 656(1), 012036.

139 S. Kausik, K. Amit and J. Pankaj, Growth and dissolution of an encapsulated contrast microbubble: effects of encapsulation permeability, Ultrasound Med. Biol., 2009, 35(8), 1385-1396.

140 C. Hernandez, L. Nieves, A. C. De Leon, R. Advincula and A. A. Exner, Role of surface tension in gas nanobubble stability under ultrasound, ACS Appl. Mater. Interfaces, 2018, 10(12), 9949-9956.

141 C. Hernandez, E. C. Abenojar, J. Hadley, A. C. De Leon, R. Coyne, R. Perera, et al., Sink or float? Characterization of shell-stabilized bulk nanobubbles using a resonant mass measurement technique, Nanoscale, 2019, 11(3), 851-855.

142 P. Nittayacharn, H. X. Yuan, C. Hernandez, P. Bielecki, H. Zhou and A. A. Exner, Enhancing tumor drug distribution with ultrasound-triggered nanobubbles, $J$. Pharm. Sci., 2019, 108(9), 3091-3098.
143 S. Xu, Z. Nie, M. Seo, P. Lewis, E. Kumacheva, H. A. Stone, et al., Generation of monodisperse particles by using microfluidics: control over size, shape, and composition, Angew. Chem., 2005, 117(5), 734-738.

144 Y. Xie, A. J. Dixon, J. M. R. Rickel, A. L. Klibanov and J. A. Hossack, Closed-loop feedback control of microbubble diameter from a flow-focusing microfluidic device, Biomicrofluidics, 2020, 14(3), 034101.

145 E. Talu, M. M. Lozano, R. L. Powell, P. A. Dayton and M. L. Longo, Long-term stability by lipid coating monodisperse microbubbles formed by a flow-focusing device, Langmuir, 2006, 22(23), 9487-9490.

146 J. H. Xu, S. W. Li, Y. J. Wang and G. S. Luo, Controllable gasliquid phase flow patterns and monodisperse microbubbles in a microfluidic T-junction device, Appl. Phys. Lett., 2006, 88(13), 2004-2007.

147 J. Jin, R. Wang, J. Tang, L. Yang, Z. Feng, C. Xu, et al., Dynamic tracking of bulk nanobubbles from microbubbles shrinkage to collapse, Colloids Surf., A, 2020, 589, 124430.

148 S. A. Peyman, J. R. McLaughlan, R. H. Abou-Saleh, G. Marston, B. R. G. Johnson, S. Freear, et al., On-chip preparation of nanoscale contrast agents towards highresolution ultrasound imaging, Lab Chip, 2016, 16(4), 679-687.

149 J. Xu, A. Salari, Y. Wang, X. He, L. Kerr, A. Darbandi, et al., Microfluidic Generation of Monodisperse Nanobubbles by Selective Gas Dissolution, Small, 2021, 2100345, 1-12.

150 N. Dietrich, S. Poncin, N. Midoux and H. Z. Li, Bubble formation dynamics in various flow-focusing microdevices, Langmuir, 2008, 24(24), 13904-13911.

151 T. Fu, Y. Ma, D. Funfschilling and H. Z. Li, Bubble formation and breakup mechanism in a microfluidic flow-focusing device, Chem. Eng. Sci., 2009, 64(10), 23922400 .

152 W. van Hoeve, B. Dollet, M. Versluis and D. Lohse, Microbubble formation and pinch-off scaling exponent in flow-focusing devices, Phys. Fluids, 2011, 23(9), 092001.

153 E. Talu, K. Hettiarachchi, R. L. Powell, A. P. Lee, P. A. Dayton and M. L. Longo, Maintaining monodispersity in a microbubble population formed by flow-focusing, Langmuir, 2008, 24(5), 1745-1749.

154 S. L. Anna and H. C. Mayer, Microscale tipstreaming in a microfluidic flow focusing device, Phys. Fluids, 2006, 18(12), 121512.

155 B. Dollet, W. Van Hoeve, J. P. Raven, P. Marmottant and M. Versluis, Role of the channel geometry on the bubble pinch-off in flow-focusing devices, Phys. Rev. Lett., 2008, 100(3), 1-4.

156 P. Garstecki, M. J. Fuerstman and G. M. Whitesides, Nonlinear dynamics of a flow-focusing bubble generator: an inverted dripping faucet, Phys. Rev. Lett., 2005, 94(23), 38-41.

157 S. Wang, A. H. Dhanaliwala, J. L. Chen and J. A. Hossack, Production rate and diameter analysis of spherical monodisperse microbubbles from two-dimensional, 
expanding-nozzle flow-focusing microfluidic devices, Biomicrofluidics, 2013, 7(1), 014103.

158 S. Wang, A. H. Dhanaliwala, J. L. Chen and J. A. Hossack, Optimal microbubble production rate from a flowfocusing microfluidic device as a function of nozzle size, gas pressure, and liquid flow rate, IEEE Int. Ultrason. Symp., 2011, 164-167.

159 T. Segers, E. Gaud, G. Casqueiro, A. Lassus, M. Versluis and P. Frinking, Foam-free monodisperse lipid-coated ultrasound contrast agent synthesis by flow-focusing through multi-gas-component microbubble stabilization, Appl. Phys. Lett., 2020, 116(17), 173701.

160 S. Wang, A. H. Dhanaliwala and J. A. Hossack, Modeling microbubble production rates from expanding nozzle flow-focusing microfluidic devices, IEEE Int. Ultrason. Symp., 2012, 2(1), 667-670.
$161 \mathrm{M}$. W. Weber and R. Shandas, Computational fluid dynamics analysis of microbubble formation in microfluidic flow-focusing devices, Microfluid. Nanofluidics, 2007, 3(2), 195-206.

162 P. Garstecki, A. M. Gañán-Calvo and G. M. Whitesides, Formation of bubbles and droplets in microfluidic systems, Bull. Pol. Acad. Sci. Tech. Sci., 2005, 53(4), 361-372.

163 J. Parasuraman, A. Summanwar, F. Marty, P. Basset, D. E. Angelescu and T. Bourouina, Deep reactive ion etching of sub-micrometer trenches with ultra high aspect ratio, Microelectron. Eng., 2014, 113, 35-39.

164 L. Y. Yeo, H. C. Chang, P. P. Y. Chan and J. R. Friend, Microfluidic devices for bioapplications, Small, 2011, 7(1), 12-48.

$165 \mathrm{~J}$. Friend and L. Yeo, Fabrication of microfluidic devices using polydimethylsiloxane, Biomicrofluidics, 2010, 4(2), 1-5. 